\title{
DISCLAIMER
}

This report was prepared as an account of work sponsored by an agency of the United States

Government. Neither the United States covernment nor any agency thereof, nor any of their employees, makes any warranty, express or implied, or assumes any legal liability or responsibility for the accuracy. completeness, or usefulness of any information, apparatus, product, or process disclosed, or represerts that its use would not infringe privately owned rights. Reference herein to any specific commercial product, jrocess, or service by trade name, trademark, manufacturer, or otherwise does not necessarily constitute or impl; its endorsement, recommendation, or favoring by the United States Government or any agency thereof. The views and opinions of authors expressed herein do not necessarily state or reflect those of the United States Government or any agency thereof.

\section{Dynamic Aperture \& Extraction Studies for the SSC High-Energy Booster}

A. W. Chao, S. K. Dutt, D. E. Johnson, T. Sen, \& Y. Yan Superconducting Super Collider Laboratory * 2550 Beckleymeade Avenue, Dallas, Texas 75237

September, 1990

- Pperated by the Universities Research Association, Inc, for the U. S. Department of Energy under Contract No. DE- 1 C02-89 2840486. 


\section{Introduction}

The final booster in the injector chain for the Superconducting Super Collider is a machine approximately twice the size of the Tevatron. Its design includes approximately $450,15+\mathrm{m}$ superconducting dipoles. The original designs specified dipoles with a $7 \mathrm{~cm}$ coil-winding diameter and an inner horizontal beam-pipe aperture of $55 \mathrm{~mm}$. 'This dipole design was chosen in order to provide an adequately large good-field aperture for both the beam injection process and for the slow-extraction of high-energy test beams. With the recent decision to increase the Collider dipole coil-winding diameter to $5 \mathrm{~cm}$, the question of the needed HEB aperture was raised. An argument for dipole commonality between the $\mathrm{HEB}$ and Collider was developed, and a preliminary examination of a $5 \mathrm{~cm} \mathrm{HEB}$ dipole was undertaken. This paper reports the results of a detailed study of the injection dynamic aperture for magnet errors corresponding to both a $5 \mathrm{~cm}$ and a $7 \mathrm{~cm}$ dipole. Also studied and reported are preliminary results of the resonant-extraction process for the two magnet designs in question. These studies are in the form of multiparticle computer simulations. The results of the studies indicate that the $7 \mathrm{~cm}$ dipole design is consistent with the desired performance requirements for the $\mathrm{HEB}$, while the $5 \mathrm{~cm}$ dipole design is marginal. We have not studied intermediate aperture values.

\section{Lattice Description}

The High Energy Booster lattice is designed to operate from $200 \mathrm{GeV}$ to $2 \mathrm{TeV}$. The design was primarily determined by the maximum energy, the need to operate in a bipolar manner, the desire to eject beams for transfer into both Collider rings from one straight section, a geometry compatible with easy injection from the Medium Energy Booster, and the expected need to have a clean, resonant extraction system to produce slow-spill test beams.

The overall design consists of two, nearly circular arcs connected by long-straight sections. The HEB geometry is shown in Fig. 1. The west long-straight section contains the ejection channels for the transfers into the collider and the extraction channel for test beams. The east straight section contains the electrostatic septa needed for the resonantextraction system. The arcs are nearly circular and each contain two shorter straight sections. These straight sections are used for the beam injection from the MEB and for 
the beam aborts. Lattice functions for one-half of the $I I F B$ are plotted in Fig. 2.

The arcs are made up of normal FODO cells with two dispersion-suppressing cells on either end. The cells have a phase advance of $90^{\circ}$ and a half length of $38.875 \mathrm{~m}$. Each half cell contains two, $15.17 \mathrm{~m}$, 6.40 $\mathrm{T}$ dipoles (at $2 \mathrm{TeV}$ ) separated by $0.65 \mathrm{~m}$, one, $1.20 \mathrm{~m}$, $200 \mathrm{~T} / \mathrm{m}$ quadrupole; and a $5.185 \mathrm{~m}$ correction spool slot length. These cells have a packing factor of $77.2 \%$ and lattice functions of $\beta_{\max }=131.8 \mathrm{~m}$ and $\eta_{\max }=3.05 \mathrm{~m}$. The dipoles in the cells are sorted on random $b_{2}$ multipnle errors and the correction elements consist of a trim dipole, a tune-adjusting quadrupole, and a chromaticitycorrecting sextupole next to the r.ll quadrupole. The straight sections have been designed to allow extraction of the beam in each direction for transfer

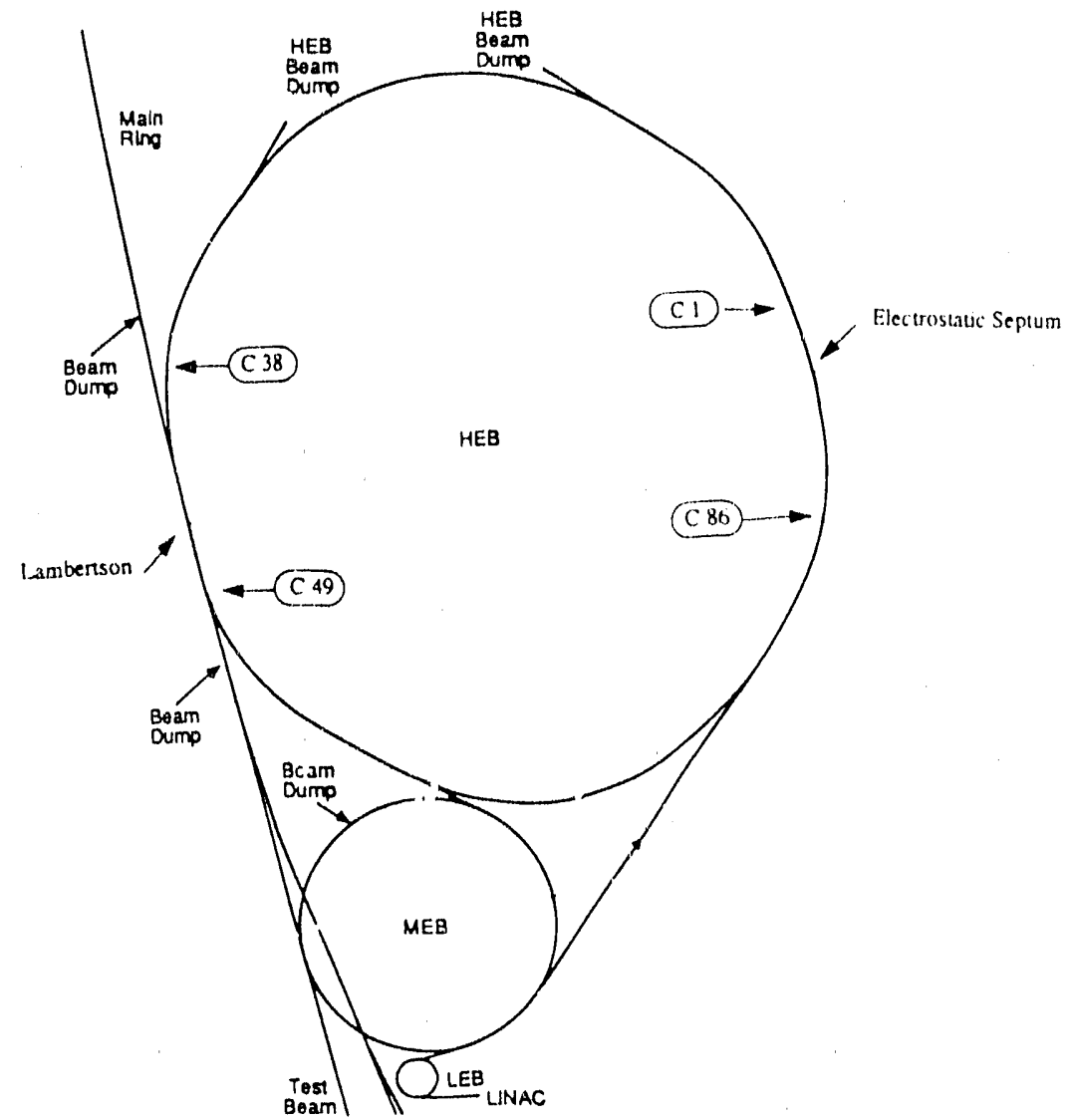

Figure 1 - Schematic plan view of the injector complex, emphasizing the functions of the six HEB straight sections. Also shown are the approximate locations of special purpose quadrupoies and octupoles in cells $1,38,49, \& 86$, used for resonant extraction.

to the Collicir, and to allow resonant extruction in one direction for test beams.

The test beams will be produced using half-integer extraction with the beam crossing an electrostatic septum on one side of the ring and then going into the extractio. channel in the opposite straight section. The extraction will take place in the horizontal plane. In order to minimize the excursions of the extracted beam in the arc magnets, the horizontal beta value in the two extraction straight sections is much larger than that of the rest of the machine. This large beta ratio allows the extracted beam to occupy the same region of the dipole aperture as that required at low energy for the injected beam. Thus, the extraction 
process should not increase the required good-field aperture. In addition, the straightsection optics can be tuned to different high-beta values at the resonant-extraction devices. A different beta value, consistent with the dipole aperture, is used while considering the extraction efficiency for the two different dipole cases under study. For the $7 \mathrm{~cm}$ dipole case, the extraction straight section was designed with $\beta_{\mathrm{H}}=305 \mathrm{~m}$. This value is related to the design of the slow extraction system at Fermilab. For the $5 \mathrm{~cm}$ dipole study, the beta value was increased to $500 \mathrm{~m}$. This choice approximately scales the extracted-beam aperture in the arcs with the good-field region of the dipoles in consideration. The beta values in the straight sections and the dipole multipole error fields are the only differences in the lattices for the two studies.

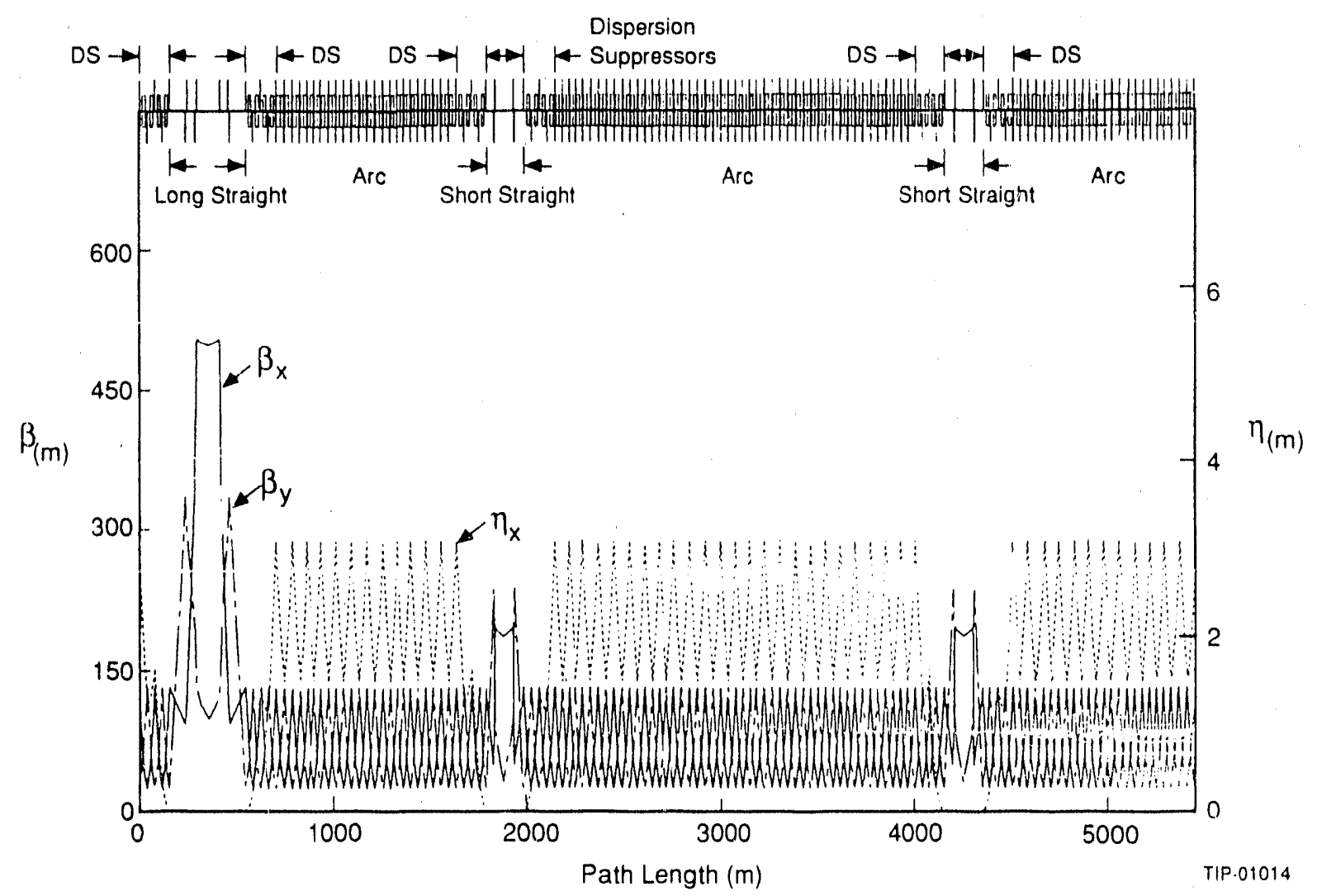

Figure 2-- Lattice functions and schematic magnet layout for one-half of the HIE ring. 
The HEB dipole good-field aperture must satisfy three different requirements for Collider injection and test-beam extraction:

- The dynamic aperture at injection must be large enough to insure that the emittance of the beam transferred into the Collider does not increase during the injection process;

- It must be large enough so that the beam required for test-beam operation does not get lost during injection; and,

- It must be adequate at maximum energy to allow the beam to circulate for several hundred turns at large amplitudes in the arcs and at a larger amplitude for the half turn immediately preceeding extraction.

For this lattice, various beam amplitudes are given below:

1. Ten sigma injection beam size $10 \sqrt{\beta \epsilon}=7.03 \mathrm{~mm}$.

2. Test beam $95 \%$ beam size at injection $\sqrt{6 \beta \epsilon_{\text {test }}+[\eta \delta]^{2}}=3.97 \mathrm{~mm}$.

3. Test beam amplitude in arcs prior to reaching the septum

$5 \mathrm{~cm}$ dipole $-13 \mathrm{~mm}$,

$7 \mathrm{~cm}$ dipole - $17 \mathrm{~mm}$.

4. Test beam $1 / 2$ turn extraction amplitude

$$
\begin{aligned}
& 5 \mathrm{~cm} \text { dipole }-20 \mathrm{~mm} \text {, } \\
& 7 \mathrm{~cm} \text { dipole }-26 \mathrm{~mm} \text {. }
\end{aligned}
$$

where the maximum amplitude in the arc is given by

$$
x_{(\max \text { in arc })}=\frac{x_{\text {sept }}+\Delta x}{\cos \theta}\left(\frac{\beta_{\text {cell }}}{\beta_{\text {sept }}}\right)^{1 / 2}
$$


and where we use a septum offset of $x_{\text {sept }} \approx 16 \mathrm{~mm}$ and a kick of $\Delta x=10 \mathrm{~mm}$, in order to obtain a linear extraction efficiency of $98 \%$. The phase angle at the septum is approximately $45^{\circ}$.

\section{Nonlinear Error Description}

SSC dipole errors have been studied in depth by Fisk, et al. ${ }^{[1]}$ Their findings, presented in the preliminary report of the magnetic errors working group, serve as: the basic reference for this section. The Fisk study considered four magnet designs, three of which were $\cos \theta$-type collared magnets, with and without cold iron, and in either a two-in-one or a one-in-one configuration. The fourth design was a superferric magnet in a one-inone configuration. Final estimates of the multipole strengths were based on a mixture of "experience and ... the calculated effects due to estimated placement errors and persistent currents in superconducting coils."[1] The dipole error multipole coefficients used in simulating the dynamic aperture as well as the extraction process are given in Table I and were obtained by scaling the values for the $4 \mathrm{~cm}$ aperture Collider dipoles given in SSC - SR -1038 , p. $84,{ }^{[2]}$ according to the laws given in $\mathrm{SSC}-7$. We enunciate these laws below. Exceptions to this scheme are also noted, along with explanatory remarks. For ease of reference, we first exhibit the definition of $b_{n}$ and $a_{n}$ used in Ref. [1], and in this report:

$$
B_{y}+i B_{x}=B_{0} \sum_{n=0}\left(b_{n}+i a_{n}\right)\left(\frac{x+i y}{r_{0}}\right)^{n},
$$

where the above symbols have their usual significance, and $r_{0}$ is a reference radius.

- The persistent current component of the systematic multipole coefficients scales inversely with the the energy.

- Let $r_{e}[4]$ denote the effective coil radius for a $4 \mathrm{~cm}$ aperture dipole, and, similarly, $r_{e}[5]$, and $r_{e}[7]$ for the $5 \mathrm{~cm}$ ani $7 \mathrm{~cm}$ aperture dipoles. The respective values are $3.0 \mathrm{~cm}, 3.5 \mathrm{~cm}$, and $4.5 \mathrm{~cm}$. Also, let $\sigma\left(b_{n}\right)[4]$ denote the rms variation of $b_{n}$ for the $4 \mathrm{~cm}$ aperture dipole, and so on. 
Table I: Multipole Coefficients for HEB Dipoles $\left[\times 10^{-4} \mathrm{~cm}^{-n}\right]$

\begin{tabular}{|c|c|c|c|c|c|c|c|c|}
\hline \multirow{3}{*}{$\begin{array}{c}\text { Multipole } \\
\text { Type }\end{array}$} & \multicolumn{6}{|c|}{ Systematic Errors } & \multirow{3}{*}{\multicolumn{2}{|c|}{ Random Errors }} \\
\hline & \multirow{2}{*}{\multicolumn{2}{|c|}{ Geometric }} & \multicolumn{4}{|c|}{ Persistent Current } & & \\
\hline & & & \multicolumn{2}{|c|}{$200 \mathrm{GeV}$} & \multicolumn{2}{|c|}{$2 \mathrm{TeV}$} & & \\
\hline \multicolumn{9}{|c|}{ Normal Multipules } \\
\hline Aperture & $5 \mathrm{~cm}$ & $7 \mathrm{~cm}$ & $5 \mathrm{~cm}$ & $7 \mathrm{~cm}$ & $5 \mathrm{~cm}$ & $7 \mathrm{~cm}$ & $5 \mathrm{~cm}$ & $7 \mathrm{~cm}$ \\
\hline$b_{1}$ & 0.15 & 0.089 & & & & & 0.56 & 0.38 \\
\hline$b_{2}$ & 0.0 & 0.0 & -1.89 & -0.89 & -0.189 & -0.089 & 0.34 & 0.18 \\
\hline$b_{3}$ & 0.054 & 0.02 & & & & & 0.18 & 0.07 \\
\hline$b_{4}$ & 0.02 & 0.02 & 0.092 & 0.026 & 0.0092 & 0.0026 & 0.35 & 0.11 \\
\hline$b_{5}$ & 0.016 & 0.0035 & & & & & 0.043 & 0.011 \\
\hline$b_{6}$ & 0.0093 & 0.0016 & -0.017 & -0.0029 & -0.0017 & -0.00029 & 0.073 & 0.014 \\
\hline$b_{7}$ & 0.029 & 0.0039 & & & & & 0.063 & 0.0096 \\
\hline$b_{8}$ & 0.009 & 0.00094 & & & & & 0.027 & 0.0032 \\
\hline \multicolumn{9}{|c|}{ Skew Multipoles } \\
\hline$a_{1}$ & 0.15 & 0.09 & & & & & 1.40 & 0.95 \\
\hline$a_{2}$ & 0.06 & 0.03 & & & & & 0.41 & 0.22 \\
\hline$a_{3}$ & 0.11 & 0.04 & & & & & 0.41 & 0.17 \\
\hline$a_{4}$ & 0.09 & 0.03 & & & & & 0.10 & 0.032 \\
\hline$a_{5}$ & & & & & & & 0.086 & 0.022 \\
\hline$a_{0}$ & & & & & & & 0.037 & 0.0072 \\
\hline$a_{7}$ & & & & & & & 0.063 & 0.0096 \\
\hline$a_{8}$ & & & & & & & 0.027 & 0.0032 \\
\hline
\end{tabular}

The scaling laws are

$$
\begin{array}{lr}
\text { Random multipoles-normal: } & \sigma\left(b_{n}\right)[5]=\sigma\left(b_{n}\right)[4]\left[\frac{r_{c}[4]}{r_{n}[5]}\right]^{n+1 / 2} \\
\text { Random multipoles-skew: } & \sigma\left(a_{n}\right)[5]=\sigma\left(a_{n}\right)[4]\left[\frac{r_{r}[4]}{r_{r}[5]}\right]^{n+1 / 2} \\
\text { Systematic multipoles-normal: } & \sigma\left(b_{n}\right)[5]=\sigma\left(b_{n}\right)[4]\left[\frac{r_{r}[4]}{r_{c}[5]}\right]^{n+1} \\
\text { Systematic multipoles-skew: } & \sigma\left(a_{n}\right)[5]=\sigma\left(a_{n}\right)[4]\left[\frac{r_{c}[4]}{r_{c}[5]}\right]^{n+1}
\end{array}
$$

The $7 \mathrm{~cm}$ aperture dipoles scale similarly.

- The values of geometric $b_{2}, b_{4}, b_{0}$, and $b_{8}$ are given for the $7 \mathrm{~cm}$ aperture dipoles. ${ }^{[3]}$ Values for the $5 \mathrm{~cm}$ dipoles are scaled down from the $7 \mathrm{~cm}$ aperture case. 
- The values of random $b_{2}$ are $1 / 4$ of the scaled values. It is assumed that the dipoles will be sorted to bring about this reduction.

- The values of random $a_{1}$ are 2.5 times larger than the scaled values to reflect the experience of magnets manufactured at HER.A. ${ }^{[4]}$

- The effective values of the systematic normal multipoles are obtained by adding together the absolute values of the geometric and persistent current contributions, and assigning the sign of the persistent current contribution to the sum. This reflects a worst case analysis of the way in which the different components would add up.

- Simulations of the long term dynamic aperture, and of extraction have been performed with values of skew and normal $a_{1}$ and $b_{1}$ set to zero. We assume that an appropriate correction scheme will be used.

\section{HEB Extraction}

The extraction process used in the HEB is of half-integer type. ${ }^{[5]}$ Special purpose octupoles are placed around the ring to excite the $69^{\text {th }}$ harmonic of the octupoles, thereby creating stable and unstable regions in phase space. In addition, special purpose quadrupoles are placed next to the octupoles. These quadrupoles control the stable phase space area, and are ramped in order to reduce the stable area to zero during the slow-extraction process. The ramp rate determines the time over which the beam is extracted, and is normally controlled by a feedback system.

- The exact disposition of the extraction quadrupoles and octupoles is determined by the need to orient the fixed points of the stable phase space area at the electrostatic septum on a line at about $45^{\circ}$ to the $\mathrm{x}$ axis. This brings about a favourable separation of the circulating and extracted bearns at the Lambertison located halfway around the ring. An important constraint is the maximum strength of the octupoles - we assume a maximum field strength of 35 kilogauss-inch at 1 inch for an individual octupole. Similar octupoles have been used for half-integer extraction at the Tevatron.

- It is essential that the extraction inefficiency be kept small in order to avoid quenching the superconducting dipoles. The inefficiency is determined by the ratio of the septum wire thickness to the step size at the electrostatic septum. The septum wire 
thickness is typically $0.1 \mathrm{~mm}$, so that the step size must be at least $5 \mathrm{~mm}$ to acheve an inefficiency of $2 \%$ or less. In the linit of zero stable phase space area, the dynamics of the particles is determined essentially by the outgoing separatrices produced by the combination of extraction quadrupoles and octupoles. One can compute the necessary strengths of the extraction elements from the requirement that the stable. area vanish, and from the desired step size at the septum.

- The extraction octupoles are deployed in groups of 4 . For a given octupole, we locate another of opposite strength at a phase advance of $\pi \nu$ around the ring. This serves to enh ince the $69^{\text {th }}$ harmonic of the octupoles, while cancelling the zero ${ }^{\text {th }}$ and the $138^{\text {th }}$ harmonics. The purpose of the two remaining octupoles in a group, also placed $\pi \nu$ apart with opposing signs, is to bring about a suitable phase for the fourier coefficient of the octupole perturbation. A total of six groups are used.

- The extraction quadrupoles are deployed in an identical manner at about the same locations as the octupoles. This cancels the zero ${ }^{\text {th }}$ harmonic of the quadrupole perturbation, so that the stopband halfwidth is determined purely by the quadrupole $69^{\text {th }}$ harmonic. Zero stable phase area is achieved by making the stopband halfwidth equal to or greater than the distance between the basal horizontal tune and the half integer closest to it ( 34.5 for the HEB).

- The disposition of the extraction elements is shown in Fig. 1. Going counterclockwise from the septum, we number successive cells as $1,2, \ldots$, for a total of 96 cells. The first group of extraction elements are shown at their approximate locations in Cells $1,38,49$, and 86 in Fig. 1. We enumerate all groups below:

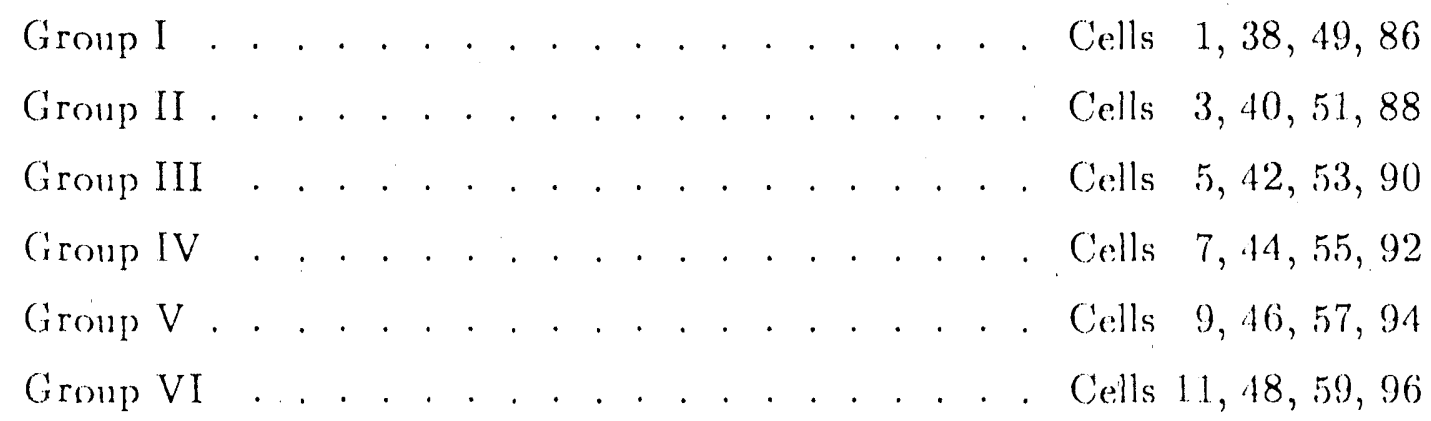

- Fig. 3 displays our preliminary results from simulating extraction in HEB lattices with both $5 \mathrm{~cm}$ and $7 \mathrm{~cm}$ dipole apertures. 'The simulations were carried out with 
500 particles initially distributed with gaussian spreads in $x, x^{\prime}, y, y^{\prime}$, and in energy. The stable phase area is set to zero from the beginning of a run (no ramping). Figs. 3(a) and 3(b) show the phase space distribution of particles after 50 turns at the electrostatic septum and the Lambertson for $\mathrm{HEB}$ optics with $5 \mathrm{~cm}$ aperture. dipoles. Figs. 3(c) and 3(d) display our results for the $7 \mathrm{~cm}$ dipole aperture case. In both cases, dipole errors have been included in the simulations ( $c f$. Table I).

\section{Conclusions About Extraction}

We preface this section with a number of remarks which will be of use in interpreting the results presented below.

- The quality of extraction can be characterized by three parameters: (i) the percentage loss suffered at the septum; (ii) the percentage loss suffered in transit between the septium and the Lambertson after a particle has been kicked; and (iii) the amount of separat ${ }^{\circ}$ in achieved between the circulating and extracted beams at the Lambertson. The loss at the septum is controlled by the wire thickness, and by the step size at that location. The transit loss is determined by the quality of the magnetic field as one moves away from the design orbit, and by the magnet aperture. The separation achieved between the circulating and extracted beams at the Lambertson is not determined simply by the kick delivered to a particle at the septum location but, rather, by the combination of the step size at the electrostatic septum, the kick administered at that point, and by the topology of the resonant phase space. This is because the dynamics of a particle is dominated by non-linear effects in this regime.

- In simulating extraction for the two HEB lattices ( $5 \mathrm{~cm}$ and $7 \mathrm{~cm}$ dipole apertures), we have not kept all parameters identical. This is because the error fields in the two cases are different ( $c f$. Table I). Thus, we have attempted, instead, to optimize the extraction process for each lattice individually. As an example of such a difference, the septum is located $16 \mathrm{~mm}$ from the design orbit for the $5 \mathrm{~cm}$ aperture case, and at $16.5 \mathrm{~mm}$ for the $7 \mathrm{~cm}$ aperture case. Also, the angular kick administered at the electrostatic septum is different in the two cases to account for the different values of the betatron amplitudes. Both cases were simulated with 5 different seeds for the random component of the dipole error coefficients, as well as 5 different seeds for the 


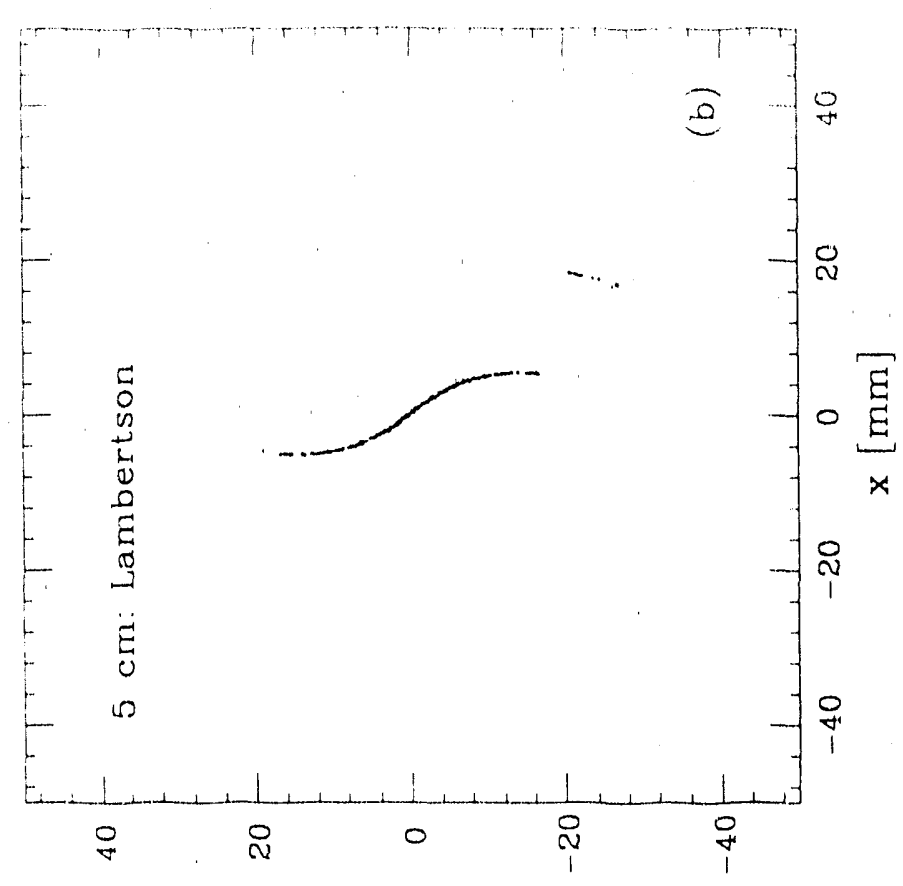

[uul] $x y+x x$

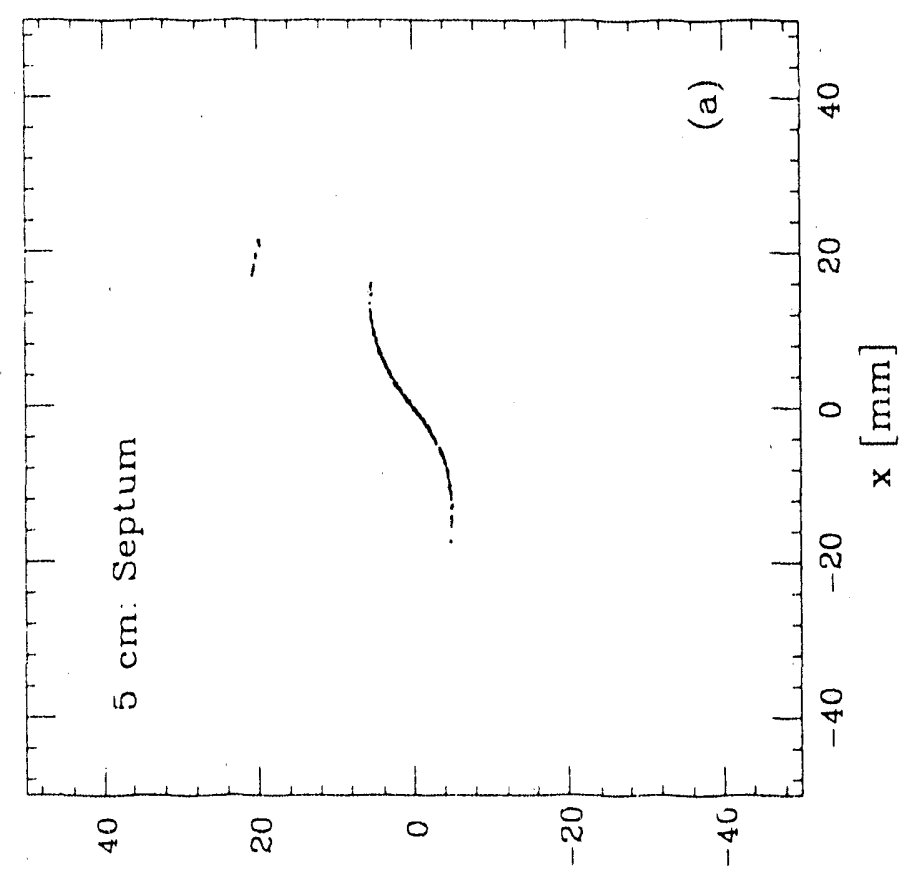

[uw] $x y+x o$

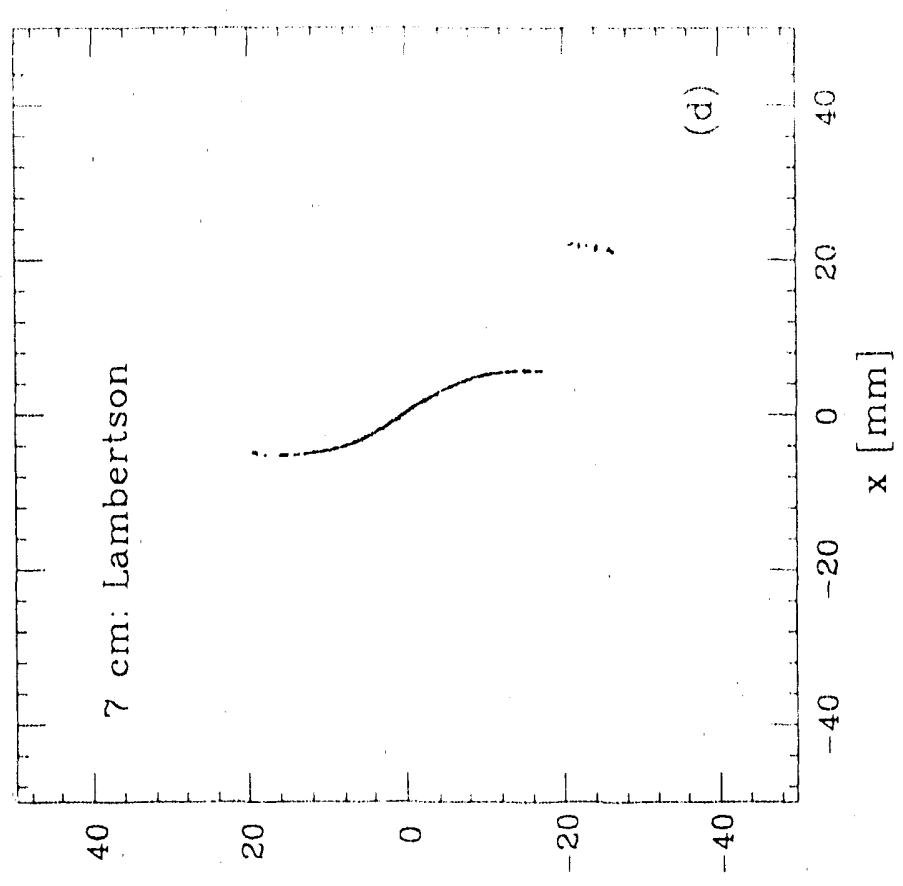

$[u[u], \times g+\times 0$

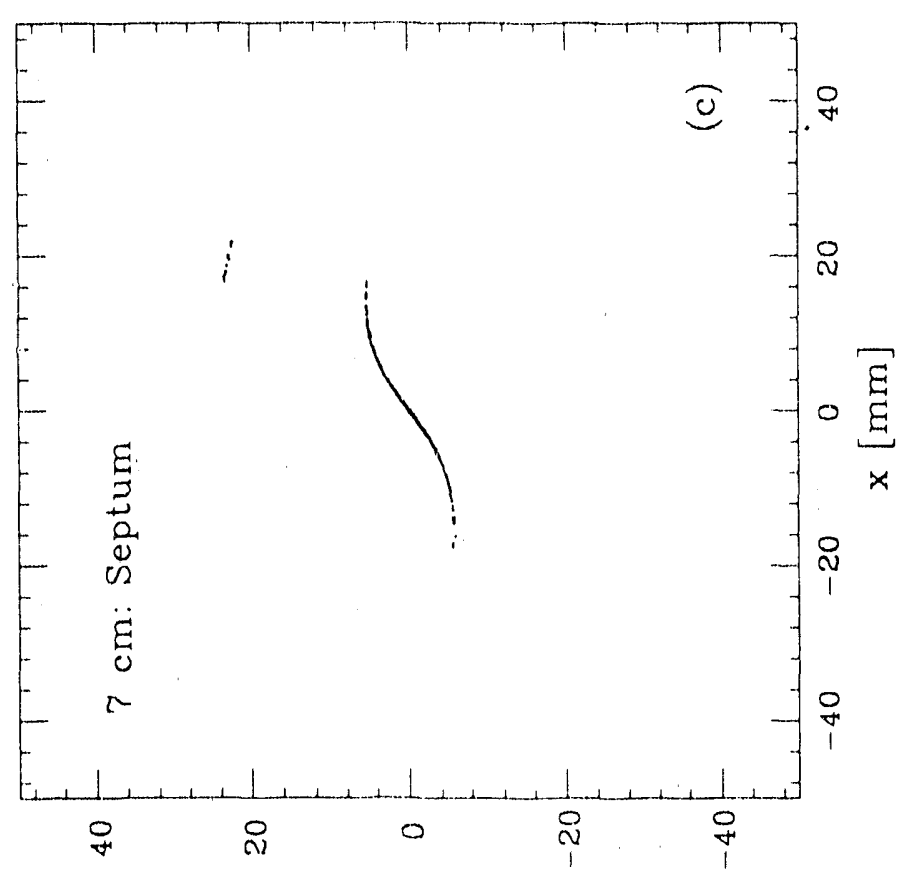

[uw], $x y+x D$

Figure 3 - Extraction simulations for the $5 \mathrm{~cm}$ and $7 \mathrm{~cm}$ dipole aperture lattices. Phatse space snapshots of the particles after 50 turns are displayed at the septum and Lambertson. 
initial phase space distribution of the particles.

- For the $5 \mathrm{~cm}$ dipole aperture, the mean inefficiency (percentage loss a.t the septum) is $2.2 \pm 0.9 \%$. No particles are lost in transit. The mean separation achieved at the Lambertson is $9.4 \pm 1.7 \mathrm{~mm}$.

- For the $7 \mathrm{~cm}$ dipole aperture, the mean inefficiency (percentage loss at the septum) is $1.8 \pm 0.6 \%$. No particles are lost in transit. 'The mean separation achieved at the Lambertson is $10.5 \pm 2.5 \mathrm{~mm}$.

On the basis of these results, one might conclude that there is only a nominal difference between the two HEB lattices as far as quality of the extraction process is concerned. However, we call attention to the phase space profiles presented in Fig. 3. A notable feature of these profiles, seen in Figs. 3(b) and 3(d), is that the tail of the circulating beam is positioned only a little beyond $5 \mathrm{~mm}$ from the design orbit. 'This may prove to be a problem in operational terms. Should it become necessary to position the tail of the circulating beam further out, to $15 \mathrm{~mm}$ or so, the two HEB lattices will behave very differently. This is because the $5 \mathrm{~cm}$ aperture lattice has stronger non-linearities, which will adversely affect the path of a kicked particle as it negotiates the final half-turn to the Lambertson. Preliminary simulations suggest that the transit loss suffered by the $5 \mathrm{~cm}$ lattice is substantially greater than that suffered by the $7 \mathrm{~cm}$ lattice, indicating the latter as the design of choice.

\section{Description of Long-term Tracking}

Long-term tracking studies were performed on Cray computers at the National Energy Research Supercomputer Center (NERSC) using a post Teapot ${ }^{[6]}$ tracking program, Ztrack. ${ }^{[7]}$ Ztrack is a vectorized code which was developed mainly for fast multi-particle tracking. It reads in a machine file and a command file. The machine file describes the linear lattice along with its non-linear elements while the command file sets up the particle. distribution, and the way particles are handled once they get lost. Usually, hundreds of particles with well distributed initial displacements are tracked simultaneously, element by element, for ten thousand turns or more. A survival plot (turn at which a particle is lost. vs. initial displacement) is then obtained for determining dynamic aperture. 
To illustrate the principle features of these simulations a brief description of the numeriral scheme is presented.

- The machine files are prepared by Teapot. A standard input (Appendix A) that describes the HEB linear lattice is prepared. Teapot reads in the in put and converts all thick elements to thin ones. All the correctors are included in the linear lattice.

- Once the linear lattice is defined, random and systematic multipole errors are added to the dipoles. Two sets of random and systematic errors, representing the $5 \mathrm{~cm}$ and $7 \mathrm{~cm}$ magnet aperture dipoles, are studied. These errors are detailed in Appendix B. There are no corrections other than the use of chromaticity sextupoles, and trim quadrupoles to modify the working tunes. (Note that the normal and skew $b_{1}$ and $a_{1}$ are set to zero.)

- After the random and systematic multipole errors are added to the lattice and the chromaticities and the tunes are adjusted to the desired values, errors due to misalignments are added. The inputs are shown in Appendix C. These errors include: (1) random steering errors $\left(\sigma_{a_{11}}\right.$ and $\left.\sigma_{t_{11}}\right),(2)$ dipole rotations $\left(\sigma_{\theta}\right)$, and dipole displacements $\left(\sigma_{x}\right.$ and $\left.\sigma_{y}\right),(3)$ quadrupole displacements, and (4) beam prosition monitor (bpm) displacements. After the addition of misalignment errors, the orbit is distorted and corrected to an rms orbit deviation of $1 . \mathrm{mm}$ in both the horizontal and vertical planes (with respect to the reference orbit).

- Once the orbit is corrected to a desired value of rms orbit deviation, a machine file is written and transferred to a Cray at NERSC where Teapot reads in the machine. file and readjusts the chromaticities and the working tunes. A machine file suitable for Ztrack is then writen.

- Ztrack reads tracking instructions from a command file. A simple example of this file is given in Appendix D. Based on the command file, Ztrack adds in an rf cavity if synchrotron oscillations are required. A Topdrawer ${ }^{[8]}$ file is written for the survival plot at the end of the tracking process. In addition, a restart file is written every 'ntsave' turns to provide a measure of protection from a communications or computer hiatus. This also enables us to retrieve information periodically from particularly long runs, or to extend a given run. 


\section{Long-term tracking results}

Long-term tracking was performed for two working tunes $\left(\nu_{x}, \nu_{y}\right)=(34.42,33.38)$ and (34.425, 33.415), each with 5 random seeds for both the $5 \mathrm{~cm}$ and the $7 \mathrm{~cm}$ coil-diameter dipole cases. Chromaticities were fitted to either 0 or 5 units. All particles were initiated with the same energy, $200 \mathrm{GeV}$ ( $\mathrm{HEB}$ injection lattice), and with a $3 \sigma_{\text {rms synchrotron }}$ oscillation amplitude, but with different transverse $\left(x / \sqrt{\beta_{x}}=y / \sqrt{\beta_{y}}\right)$ amplitudes.

Long-term tracking for the first set of working tunes $(34.42,33.38)$ was performed first: Survival plots are shown in Figs. $4-7$.
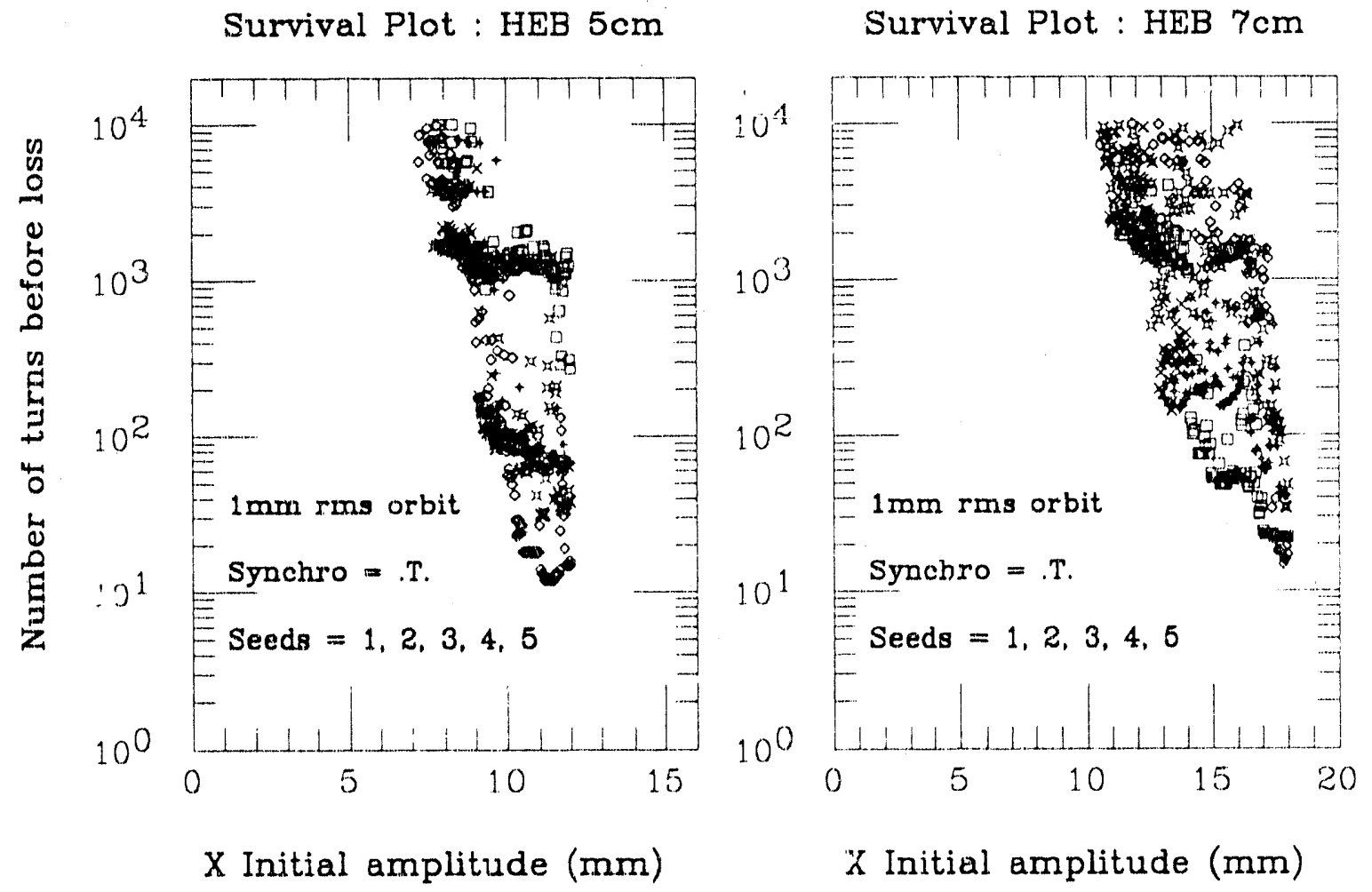

Figure $4-10,000-t u r n$ survival plots for the first set of working tunes $(34.42,33.38)$. All five seeds have zero chromaticity.

Figure 4 shows the 10,000-turn results for zero chromaticities. All 5 seeds are plotted in the same frame with different symbols. These same results are also shown in Figure, 5 with the $5 \mathrm{~cm}$ coil-diameter dipole case and the $7 \mathrm{~cm}$ coil-diameter dipole case plotted in the same frame but with 5 frames for 5 different seeds. Seed 11 was chosen for a half million 


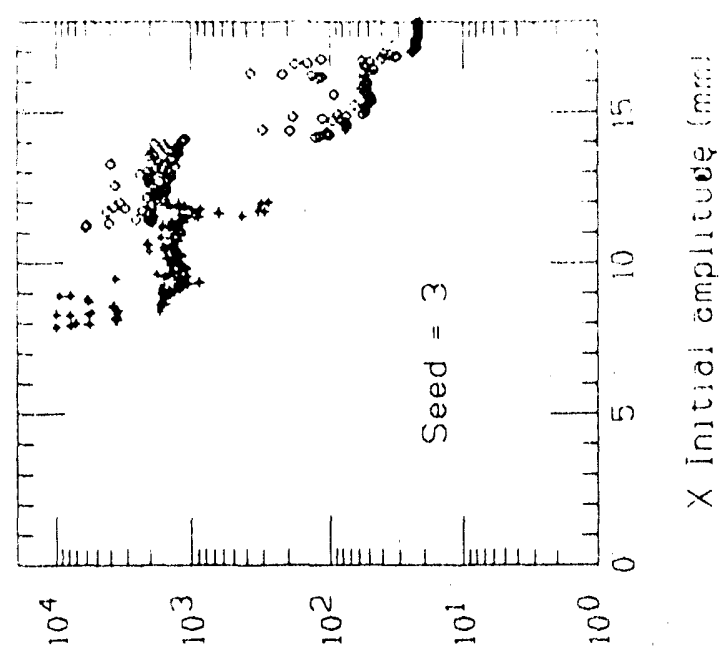

sjoi auojaq sujni jo jaqunN
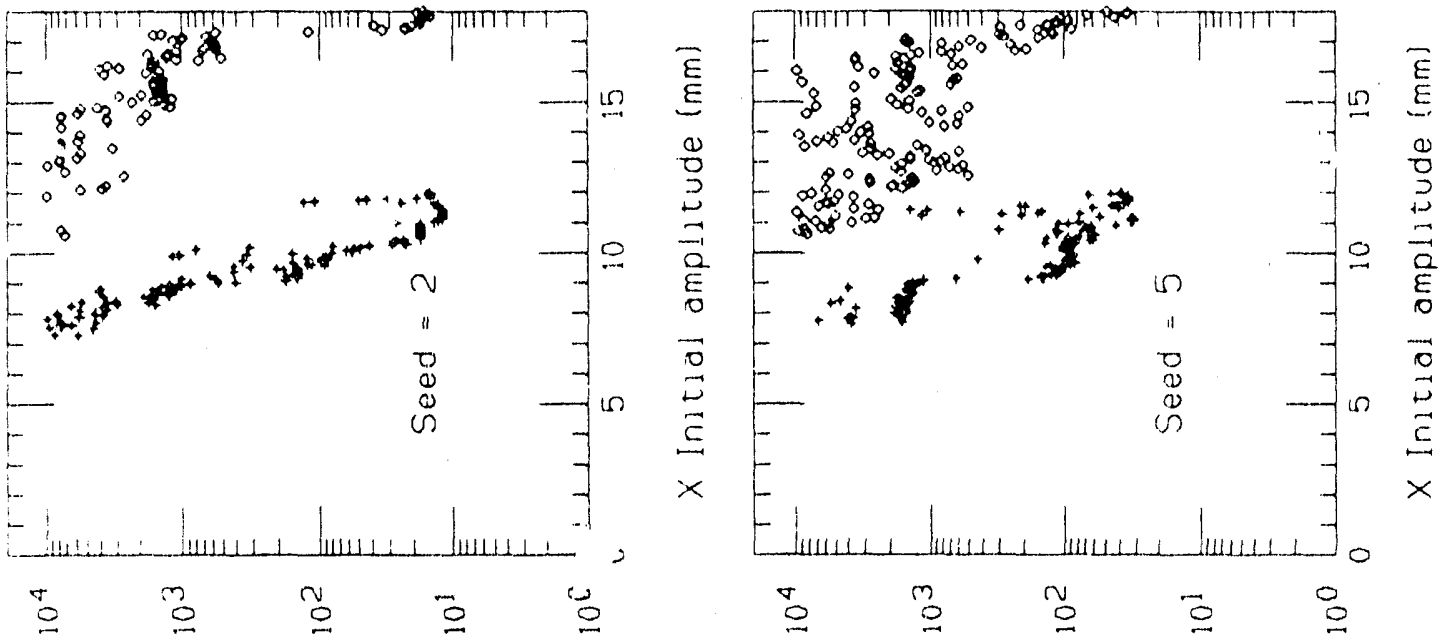

ssol ajojaq sujna jo daqunN ssot ajojaq suind fo jaqunN
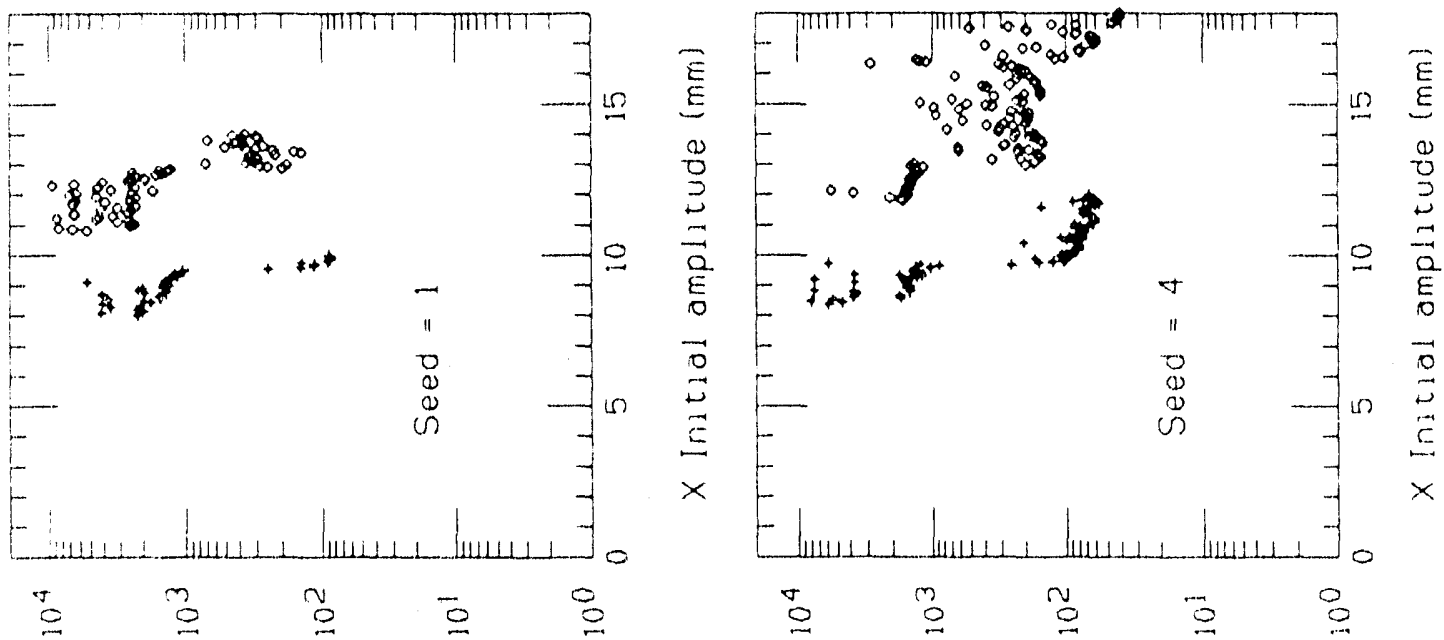

ssol a dotad sumn jo daqurin

ssol ajojag suwnz go jacunn

Figure 5 - 10,000-turn survivil plets for the first set of working tunes with zero chromaticities, comparing the 5 and $7 \mathrm{~cm}$ dipole apertures. 

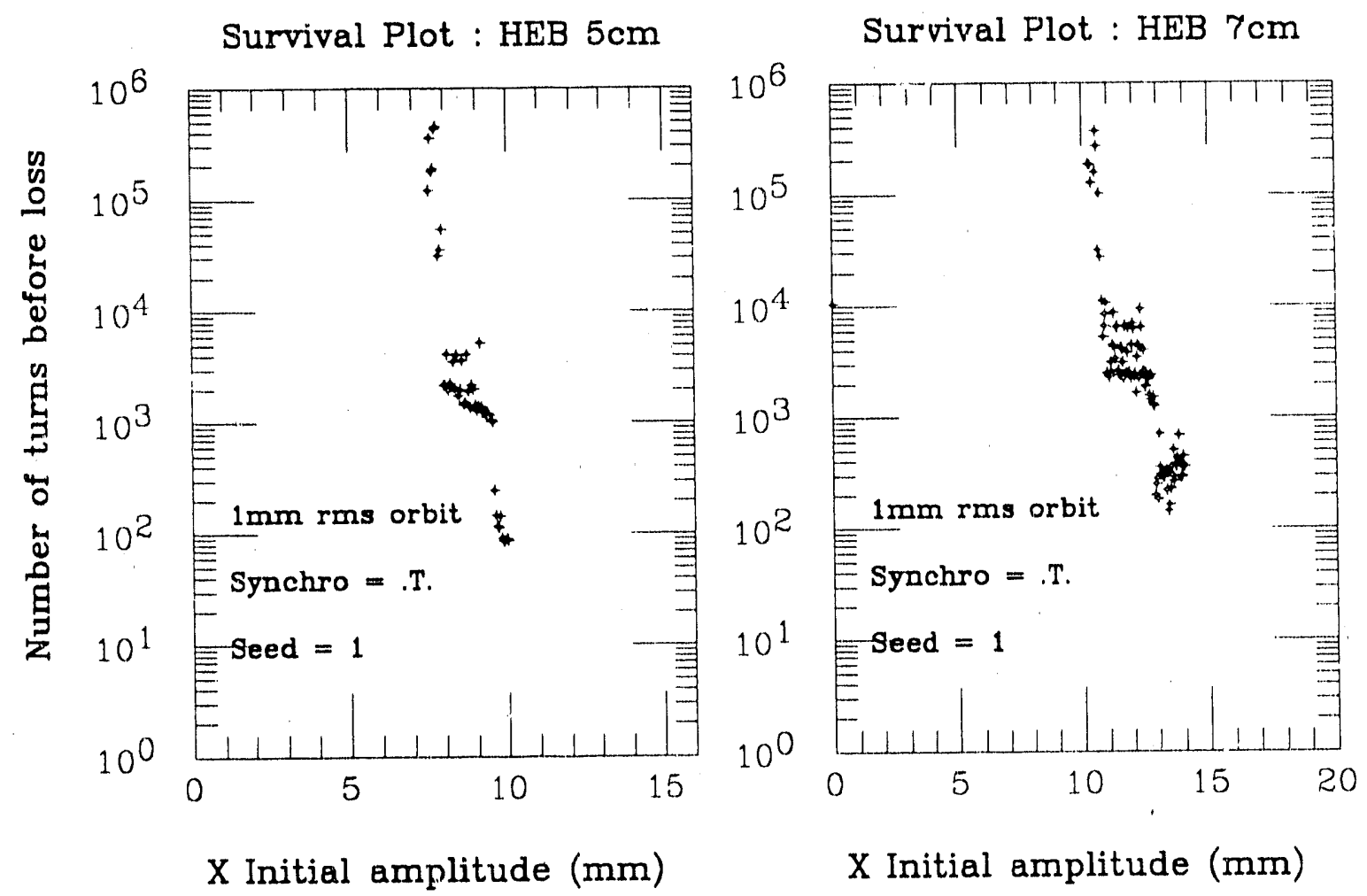

Figure $6-500,000$-turn survival plots for zero chromaticity and the first set of working tunes.

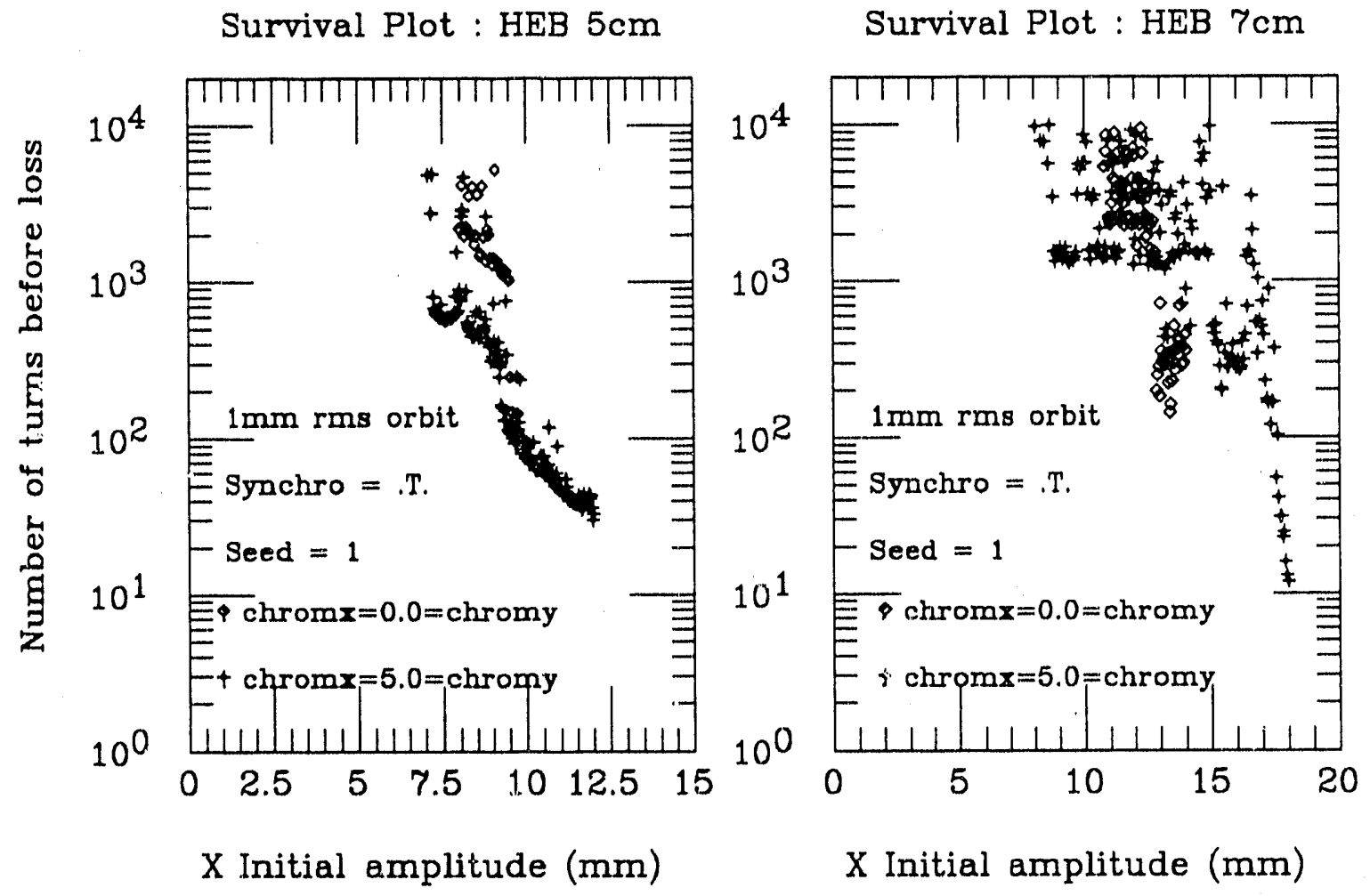

Figure 7 - 10,000-turn survival plots for the first set of tunes for 0 and 5 units of chromaticity. 
turn tracking study (the HEB fill time). The results are shown in Fig. 6(a) for the $5 \mathrm{~cm}$ coil-diameter dipole case and in $6(\mathrm{~b})$ for the $7 \mathrm{~cm}$ coil-diameter dipole case. The results of fitting the chromaticities to 5 units for Seed \#1 are shown in Fig. 7(a) for the $5 \mathrm{~cm}$ coil-diameter dipole case and Fig. $7(\mathrm{~b})$ for the $7 \mathrm{~cm}$ coil-diameter dipole case. Two sets of data, one for 5 units of chromaticity and the other for zero chromaticity are plotted in each frame with different symbols. The dynamic apertures are significantly smaller for the 5 unit chromaticity cases. Such an effect was diagnosed as an inappropriate choice of the working tunes.

Survival plots for the tunes $(34.425,33.415)$ are shown in Figs. $8-11$.

Survival Plot : HEB $5 \mathrm{~cm}$

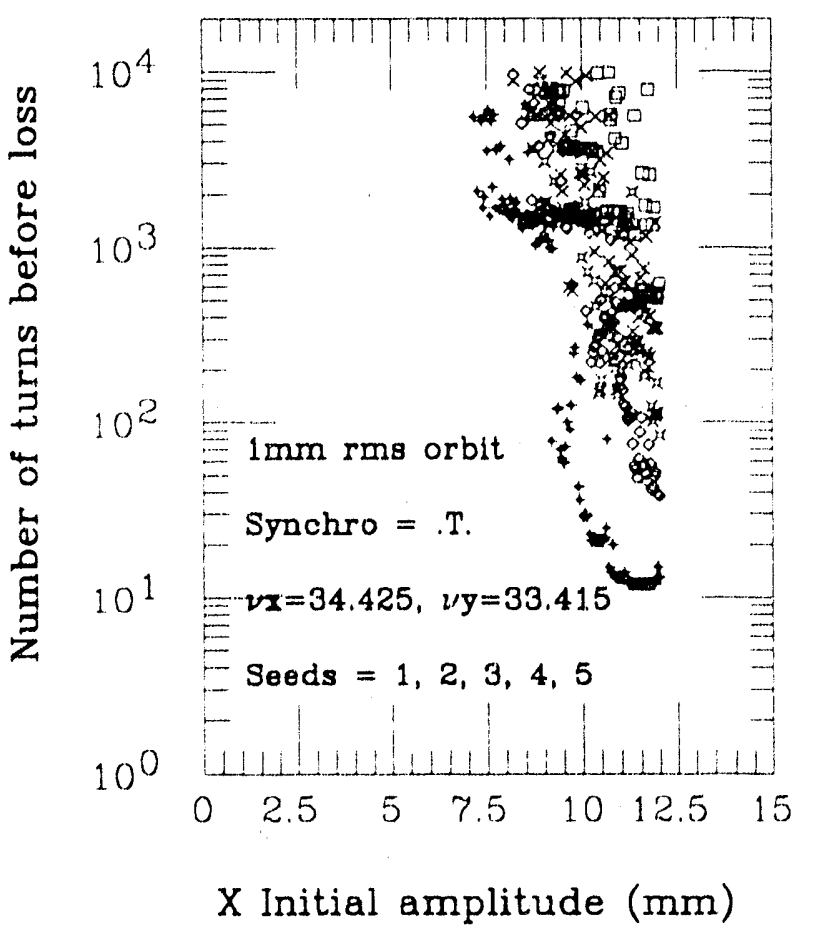

Survival Plot : HEB $7 \mathrm{~cm}$

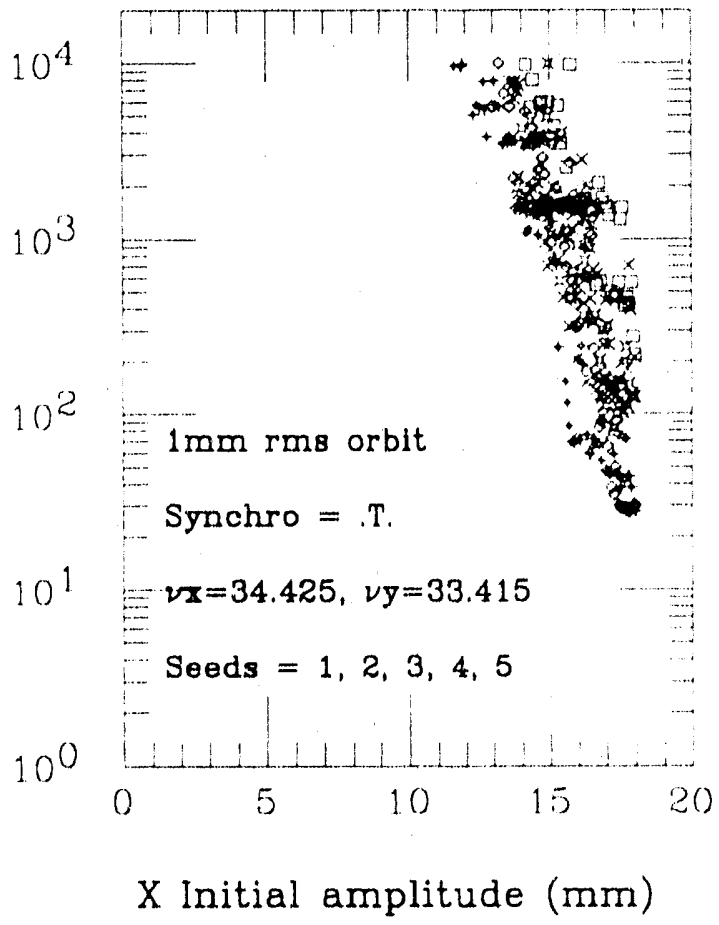

Figure $8-10,000$ turn survival plots for the tunes $(34.425,33.415)$ with zero chromaticity. 
Figs. 8 \& 9 show the 10,000 -turn results for the 0 and 5 unit chromaticity cases respectively. Each figure has two frames, one for the $5 \mathrm{~cm}$ coil-diameter dipole case and the other for the $7 \mathrm{~cm}$ coil-diameter dipole case. In each frame, the data from each of the 5 seeds are plotted with different symbols.

The 5 unit chromaticity effect is negligible and the dynamic apertures are as large as the previous cases with working tunes set at $(34.42,33.38)$. 500,000 turn tracking studies were also performed for this set of tunes. The results are shown in Figs. 10 \& 11 for the 0 and 5 unit chromaticity cases respectively. The dynamic apertures are slightly reduced compared to the 10,000-turn dynamic apertures. Based on the above results, the $7 \mathrm{~cm}$ coil-diameter dipole has an adequate dy namic aperture for the $\mathrm{HEB}$, while the $5 \mathrm{~cm}$ coil-diameter dipole has only a marginally adequate dynamic aperture.

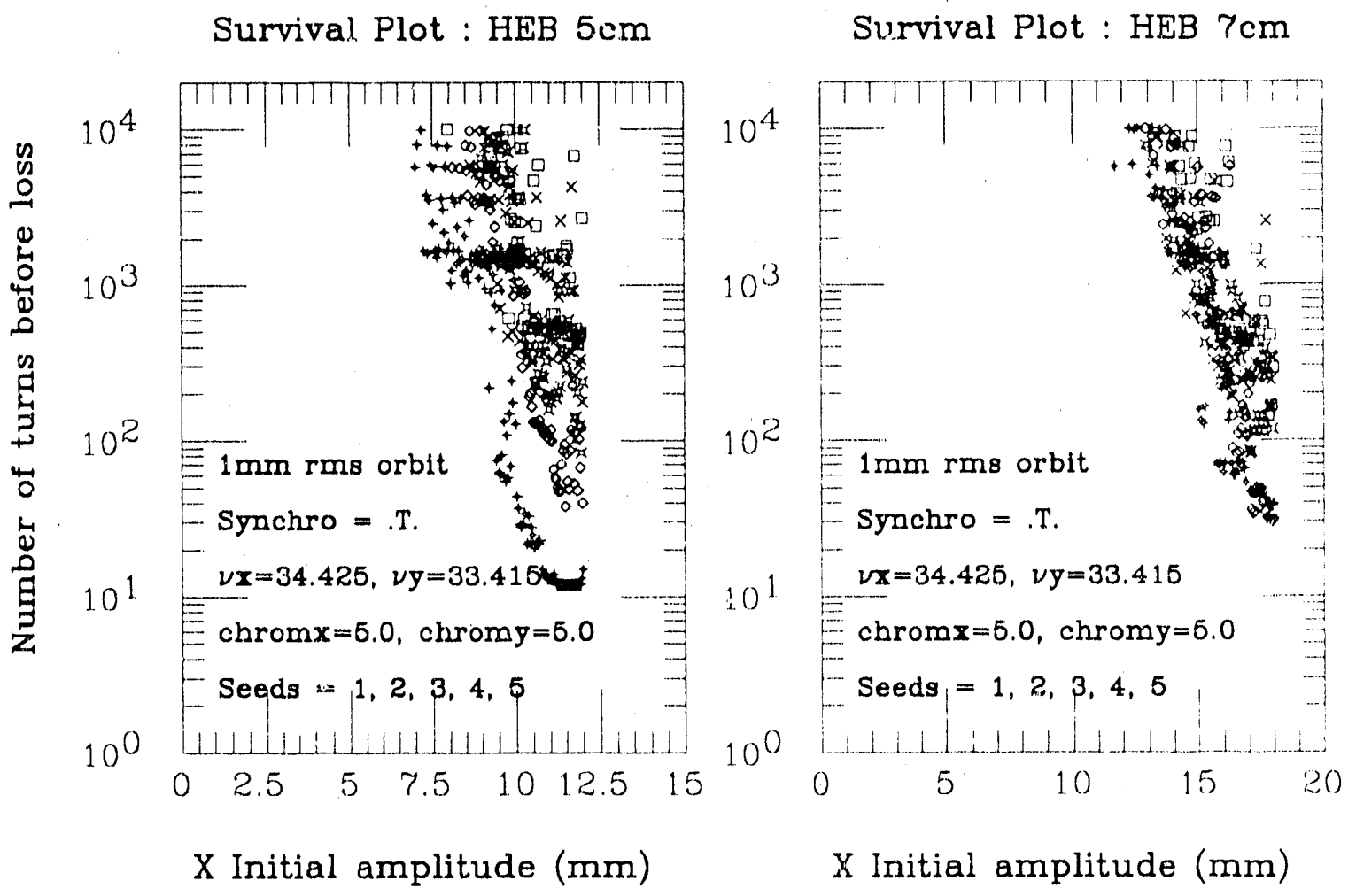

Figure 9 - 10,000-turn survival plots for the tunes $(34.425,33.415)$ with 5 units chromaticity. 
Survival Plot : HEB, $200 \mathrm{Gev}$

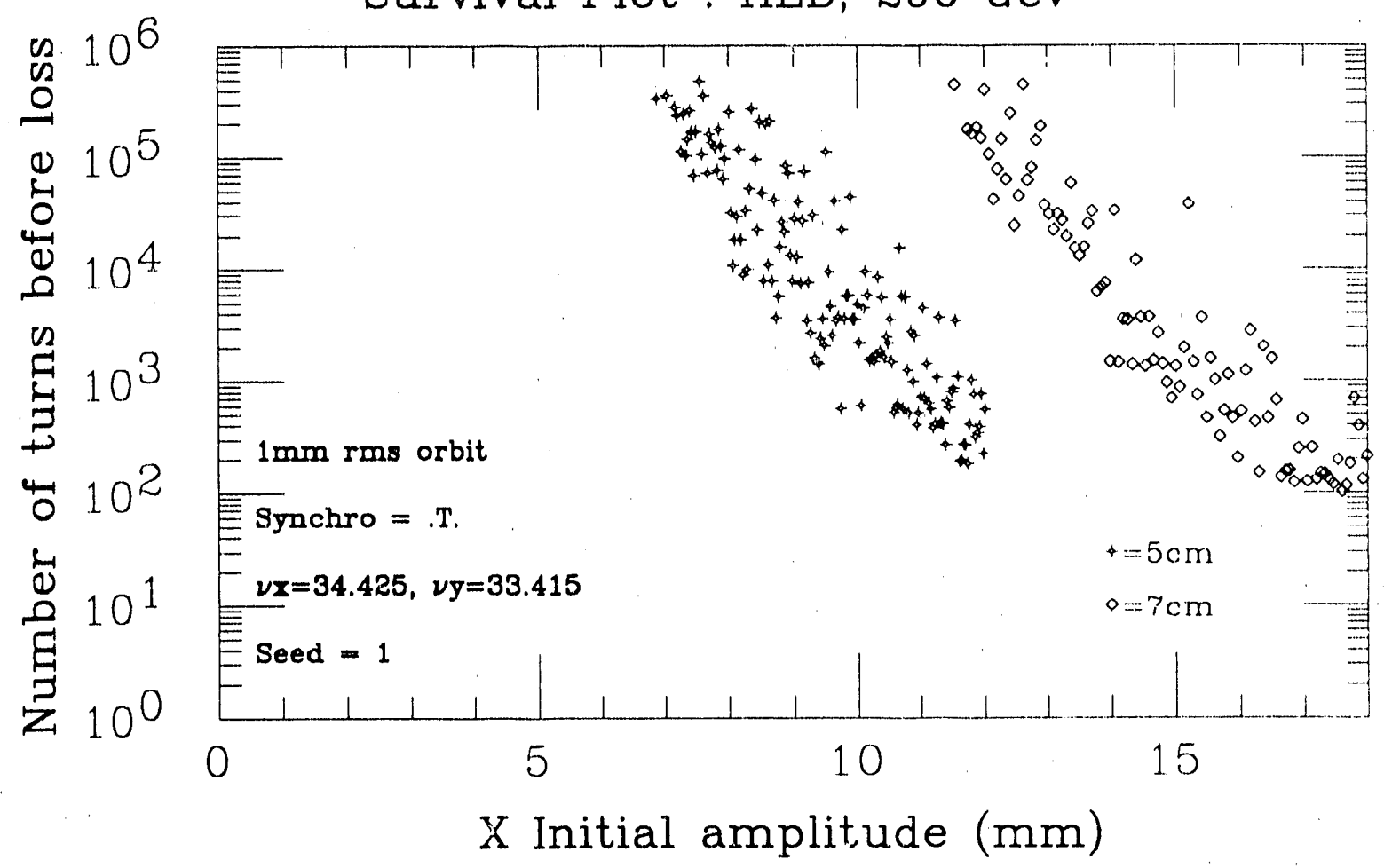

Figure $10-500,000$-turn survival plots for the tune $(34.425,33.415)$ with zero chromaticity.

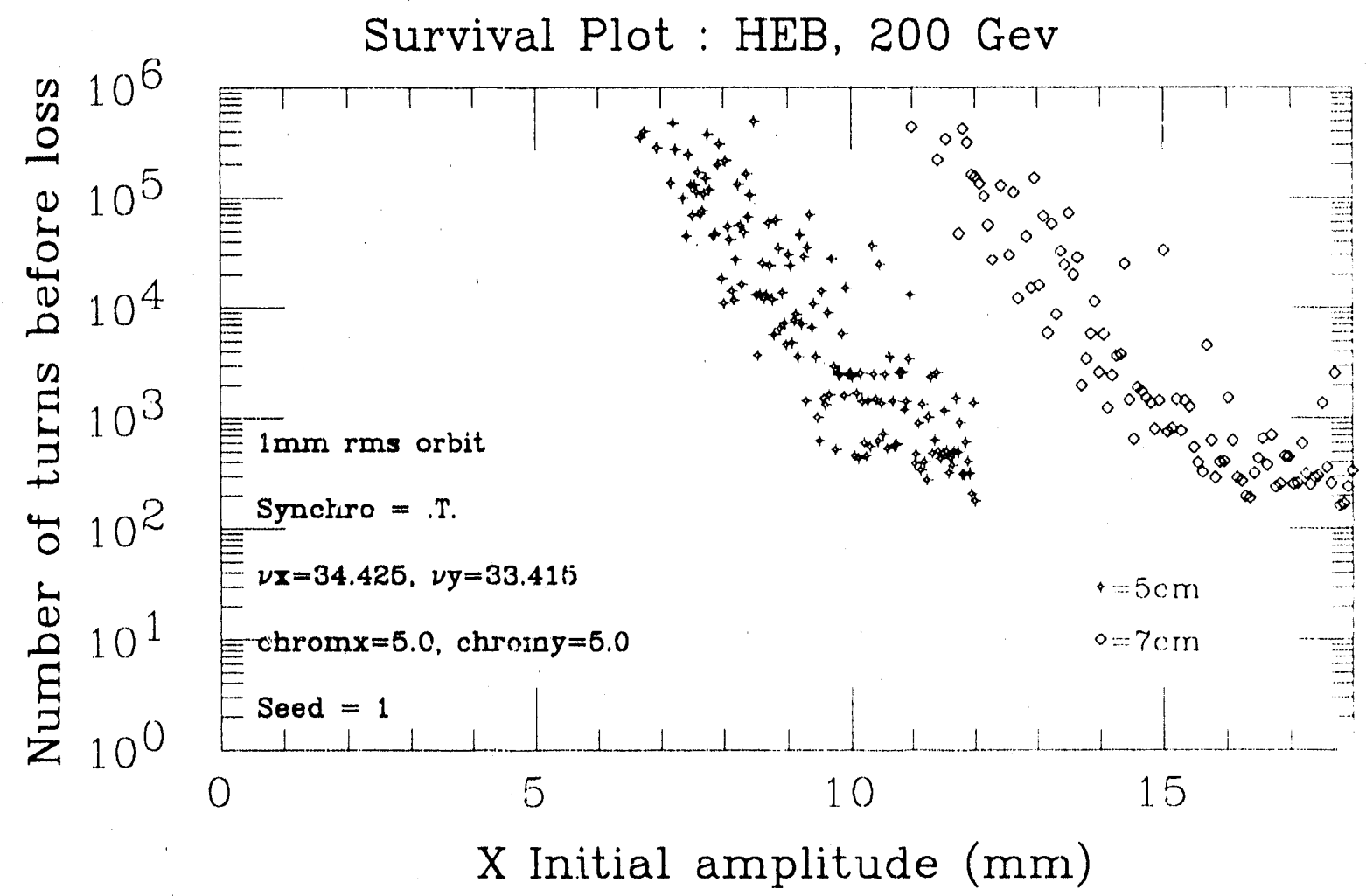

Figure $11-500,000$-turn survival plots for the tune $(34.425,33.415)$ with 5 units chromaticity. 


\section{Conclusions}

The choice of the $\mathrm{HEB}$ dipole magnet aperture depends on the dynamic apertire at injection and on efficient slow extraction at $2 \mathrm{TeV}$. The present HEB program specifies a dipole with a coil-winding diameter of $5 \mathrm{~cm}$. We have made a systematic study of the adequacy of this choice and have found it to be marginal. The $7 \mathrm{~cm}$ design, on the other hand, was shown to be acceptable. We have not studied intermediate aperture choices.

In the above studies we have assumed that the dipole magnet errors (random, systematic, and persistent-current contributions) can be estimated by scaling from the expected $4 \mathrm{~cm}$ dipole design.

From the slow-extraction studies, we cari see that the resonant extraction efficiency is somewhat worse for the smaller dipole aperture case, although both the $5 \mathrm{~cm}$ and the $7 \mathrm{~cm}$ dipoles possess acceptable apertures for the extraction process. Should we need to move the inner edge of the Lambertson channel ont beyond approximately $5 \mathrm{~mm}$, however, indications are that the $5 \mathrm{~cm}$ aperture extraction efficiency would be unacceptable, while the $7 \mathrm{~cm}$ case would remain adequate.

In the dynamic aperture studies, we suggest the requirement that the dynamic aperture be larger that ten times the nominal rms betatron beam size $\left(x_{0}=y_{0}=7 \mathrm{~mm}\right)$, when the particle is executing a three-sigma synchrotron oscillation. The HEB was simulated with a $1 \mathrm{~mm}$ rms closed orbit error, but we have assumed a perfect correction of linear effects. Under these assumptions, we conclude that the $5 \mathrm{~cm}$ design per se is not ardequate. To conclude otherwise would require, for example, a better working point, a simple and effective correction scheme, or a good reason to reduce the aperture goal mentioned above. Subsequent studies should concentrate on these areas. In the event that these concerns are not adequately resolved it may be necessary to increase the dipole apertiare from the envisioned $5 \mathrm{~cm}$ 


\section{References}

1. H. E. Fisk, et al., "Preliminary Report of the Magnetic-Errors Working Group of the SSC Aperture Workshop", SSC-7 (1985).

2. D. Bintinger, et al., "Compensation of SSC Lattice Optics in the Presence of Dipole Field Errors', SSC-SR-1038 (1989).

3. F. Harfoush, et al., "The Design of a Large Aperture High Field Dipole", Fermilab internal report (1989).

4. K. Kauffmann, T. Garavaglia, and R. Steining, "Application of the SSCTRK Numerical Simulation Program to the Evaluation of the SSC Magnet Aperture", SSCL-265 (1990).

5. D. A. Edwards, "Comparison of Half Integer and Third Integer Extraction for the Energy Doubler", Fermilab note (1978).

6. L. Schachinger and R. Talman, "TEAPOT. A Thin Element Accelerator Program for Optics and Tracking", SSC-52 (1985).

7. Y. Yan, "Supercomputing for the Superconducting Super Collider", SSCL-239 (1990).

8. R. B. Chaffee, "Top Drawer", SLAC Computation Group Note CGTM No. 178 (1980). 


\section{Appendix A: Linear Lattice Data}

TITLE

BPM :

MONITOR, $L=0.0$

$\mathrm{QF}$ :

QUADROPOLE, $L=0.87500000000000, \mathrm{~K} 1=0.02110750000000, \&$ TYPE = IR

01

DRIFT, $L=11.85865223800000$

B :

SBEND, $L=7.50000000000000$, ANGLE $=0.00727220521516$

0:

DRIFT, $L=0.65000000000000$

001:

DRIFT, $L=9.61634776200000$

QD :

QUADRUPOLE, $\mathrm{L}=0.87500000000000, \mathrm{~K} 1=-0.02110750000000, \&$ TYPE $=I R$

02:

DRIFI, $L=13.33447224700000$

002 :

DRIFT, $L=8.14052775300000$

03:

DRIFT, $I_{1}=11.77706076700000$

003 :

DRIFT, $L=9.69793923300000$

04:

DRIFT, $L=12.58576729000000$

004:

DRIFT, $L=8.88923271000000$

Q1:

QUADRUPOLE, $\mathrm{L}=0.50000000000000, \mathrm{~K} 1=0.01068115559620, \&$ TYPE $=I R$

D.1 : $\quad$ DRIFT, $L=80.07859999999999$

Q2: QUADRUPOLE, $\mathrm{L}=1.09375000000000, \mathrm{~K} 1=-0.00921884293332, \&$ TYPE = IR

D2: $\quad$ DRIFT, $L=5.00000000000000$

Q3: QUADRUPOLE, $L=1.52400000000000, K 1=0.00139832196268, \&$ TYPE $=I R$

D3: $\quad$ DRIFT, $L=36.57880000000000$

Q4: QUADROPOLE, $L=1.09375000000000, K 1=-0.00870621281946, \&$ $T Y P E=I R$

D4 :

DRIFT, $L=5.25390000000000$ 


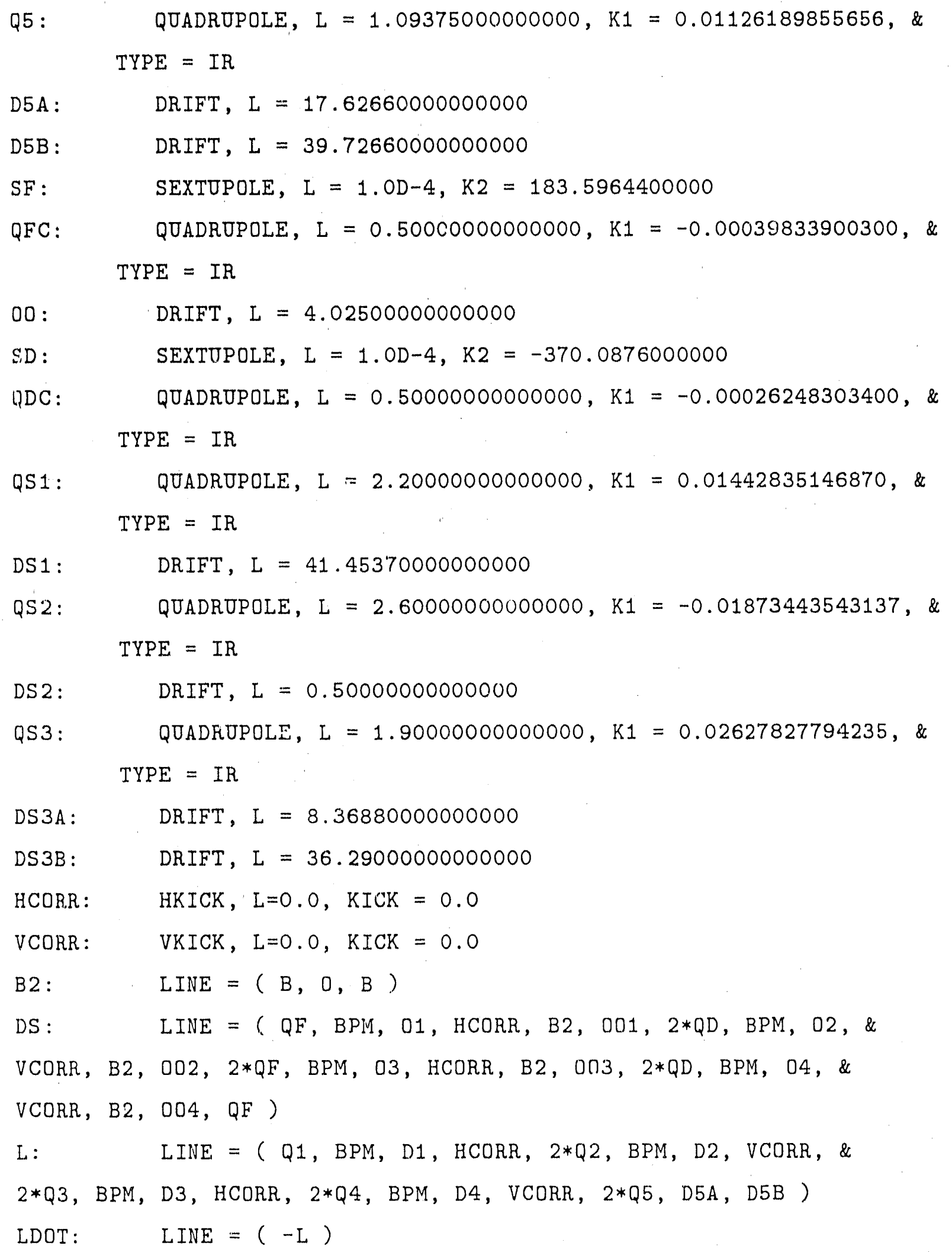




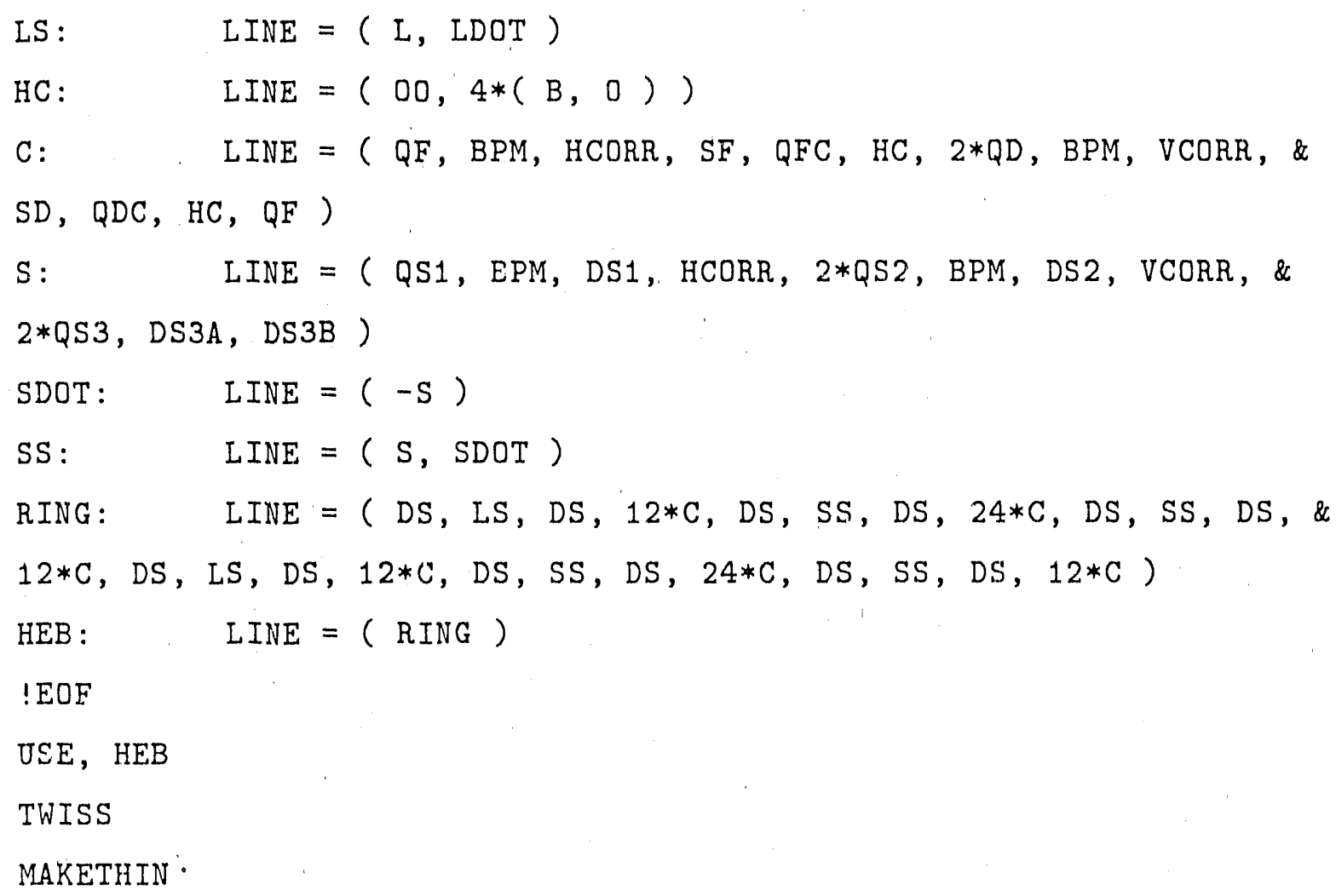




\section{Appendix B: Random and Systematic Errors}

! Put in dipole errors for the $5 \mathrm{~cm}$ coil dipoles->

! Random Errors

ERRORS, B, \&

$$
\begin{aligned}
& \text { siga1 }=0.00 \mathrm{E}+00, \quad \text { sigb1 }=0.00 \mathrm{E}+00, \quad \text { \& } \\
& \text { siga2 }=0.41 \mathrm{E}+00, \quad \operatorname{sigb2}=0.34 \mathrm{E}+00, \quad \& \\
& \text { siga3 }=0.41 \mathrm{E}+02, \quad \operatorname{sigb3}=0.18 \mathrm{E}+02, \quad \& \\
& \text { siga4 }=0.10 \mathrm{E}+04, \quad \operatorname{sigb} 4=0.35 \mathrm{E}+04, \quad \& \\
& \text { siga5 }=0.86 \mathrm{E}+05, \quad \text { sigb5 }=0.43 \mathrm{E}+05, \quad \& \\
& \text { siga6 }=2.37 \mathrm{E}+07, \quad \quad \operatorname{sigb6}=0.73 \mathrm{E}+07, \quad \& \\
& \text { siga7 }=0.63 \mathrm{E}+09, \quad \operatorname{sigb7}=0.63 \mathrm{E}+09, \quad \& \\
& \text { siga8 }=0.27 \mathrm{E}+11, \quad \operatorname{sigb8}=0.27 \mathrm{E}+11, \quad \text { \& } \\
& \text { seed }=X X X, \quad \text { cut }=6
\end{aligned}
$$

! Systematic Errors

\section{ERRORS, $B$, \&}

$$
\begin{aligned}
& \text { siga1 }=0.00 \mathrm{E}+00, \quad \operatorname{sigb1}=0.00 \mathrm{E}+00, \quad \& \\
& \text { siga2 }=0.60 \mathrm{E}-01, \quad \operatorname{sigb2}=-0.19 \mathrm{E}+01, \quad \& \\
& \text { siga3 }=0.11 E+02, \quad \operatorname{sigb3}=0.54 E+01, \quad \& \\
& \text { siga4 }=0.90 \mathrm{E}+03, \quad \text { sigb4 }=0.11 \mathrm{E}+04, \quad \text { \& } \\
& \text { siga5 }=0.00 \mathrm{E}+00, \quad \text { sigb5 }=0.16 \mathrm{E}+05, \quad \text { \& } \\
& \text { siga6 }=0.00 \mathrm{E}+00, \quad \text { sigb6 }=-0.26 \mathrm{E}+07, \quad \& \\
& \text { siga7 }=0.00 \mathrm{E}+00, \quad \text { sigb7 }=0.29 \mathrm{E}+09, \quad \& \\
& \text { siga } 8=0.00 \mathrm{E}+00, \quad \operatorname{sigb8}=0.90 \mathrm{E}+10, \quad \& \\
& \text { SYSTEMATIC }=1.0
\end{aligned}
$$


! Put in dipole errors for the $7 \mathrm{~cm}$ coil dipoles $\rightarrow$

\section{! Ranciom Erroxs}

ERRORS, $B$, \&

$$
\begin{array}{lll}
\text { siga1 }=0.00 \mathrm{E}+00, & \text { sigb1 }=0.00 \mathrm{E}+00, & \& \\
\text { siga2 }=0.22 \mathrm{E}+00, & \text { sigb2 }=0.18 \mathrm{E}+00, & \& \\
\text { siga3 }=0.17 \mathrm{E}+02, & \text { sigb3 }=0.70 \mathrm{E}+1,1, & \& \\
\text { siga4 }=0.32 \mathrm{E}+03, & \text { sigb4 }=0.11 \mathrm{E}+04, \quad \text { \& } \\
\text { siga5 }=0.22 \mathrm{E}+05, & \text { sigb5 }=0.11 \mathrm{E}+05, \quad \text { \& } \\
\text { siga6 }=0.72 \mathrm{E}+06, & \text { sigb6 }=0.14 \mathrm{E}+07, \quad \text { \& } \\
\text { siga7 }=0.96 \mathrm{E}+08, & \text { sigb7 }=0.96 \mathrm{E}+08, \quad \& \\
\text { siga8 }=0.32 \mathrm{E}+10, & \text { sigb8 }=0.32 \mathrm{E}+10, & \& \\
\text { seed }=\text { XXX, cut }=6 &
\end{array}
$$

! Systematic Errors

ERRORS, $B$, \&

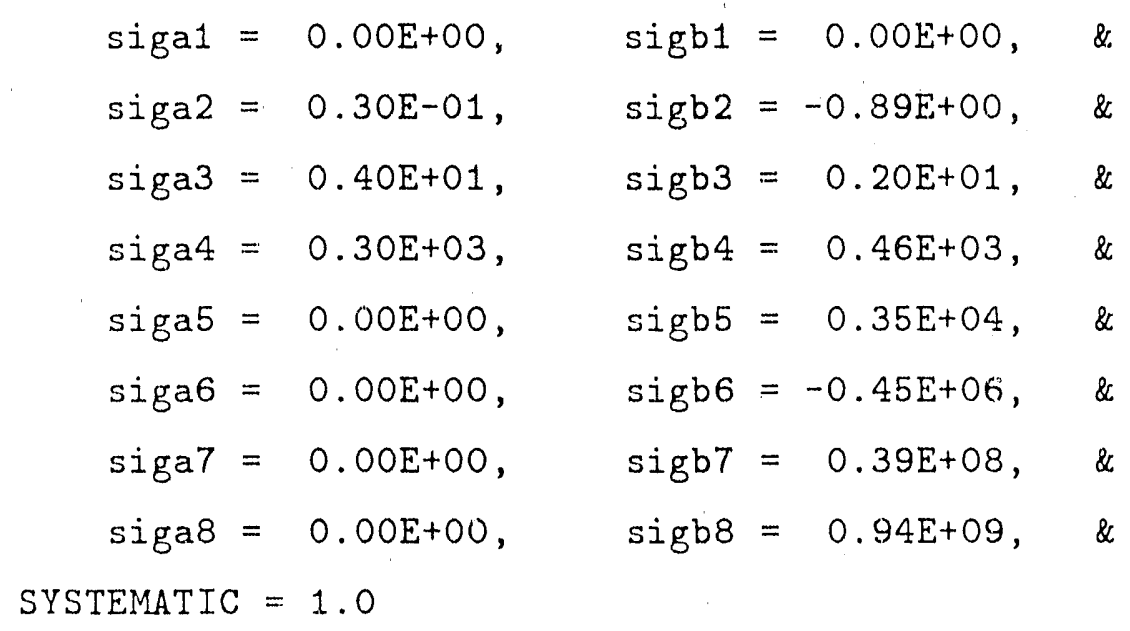


Appendix C: Misalignment Errors

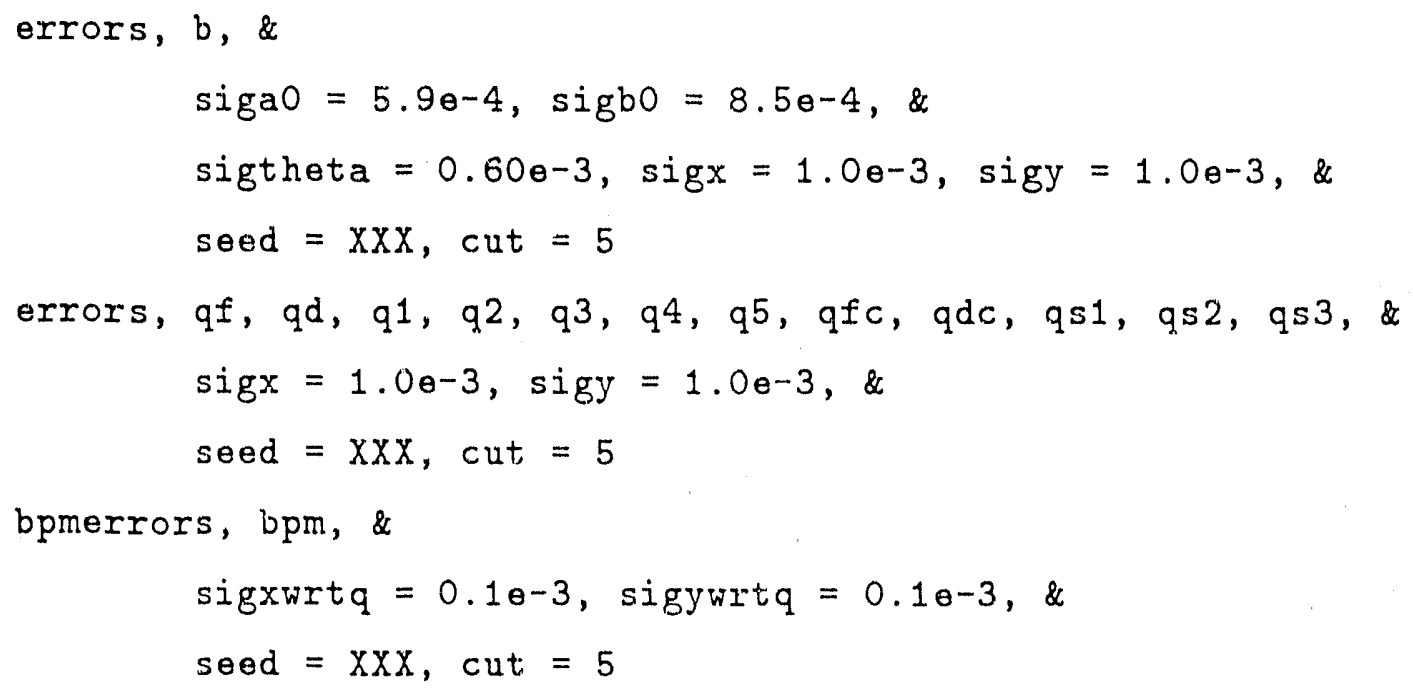


Appendix D: Namelist command file for the program "Ztrack"

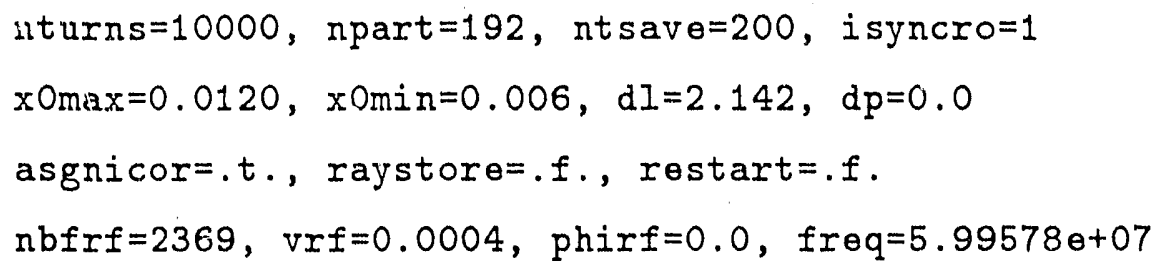



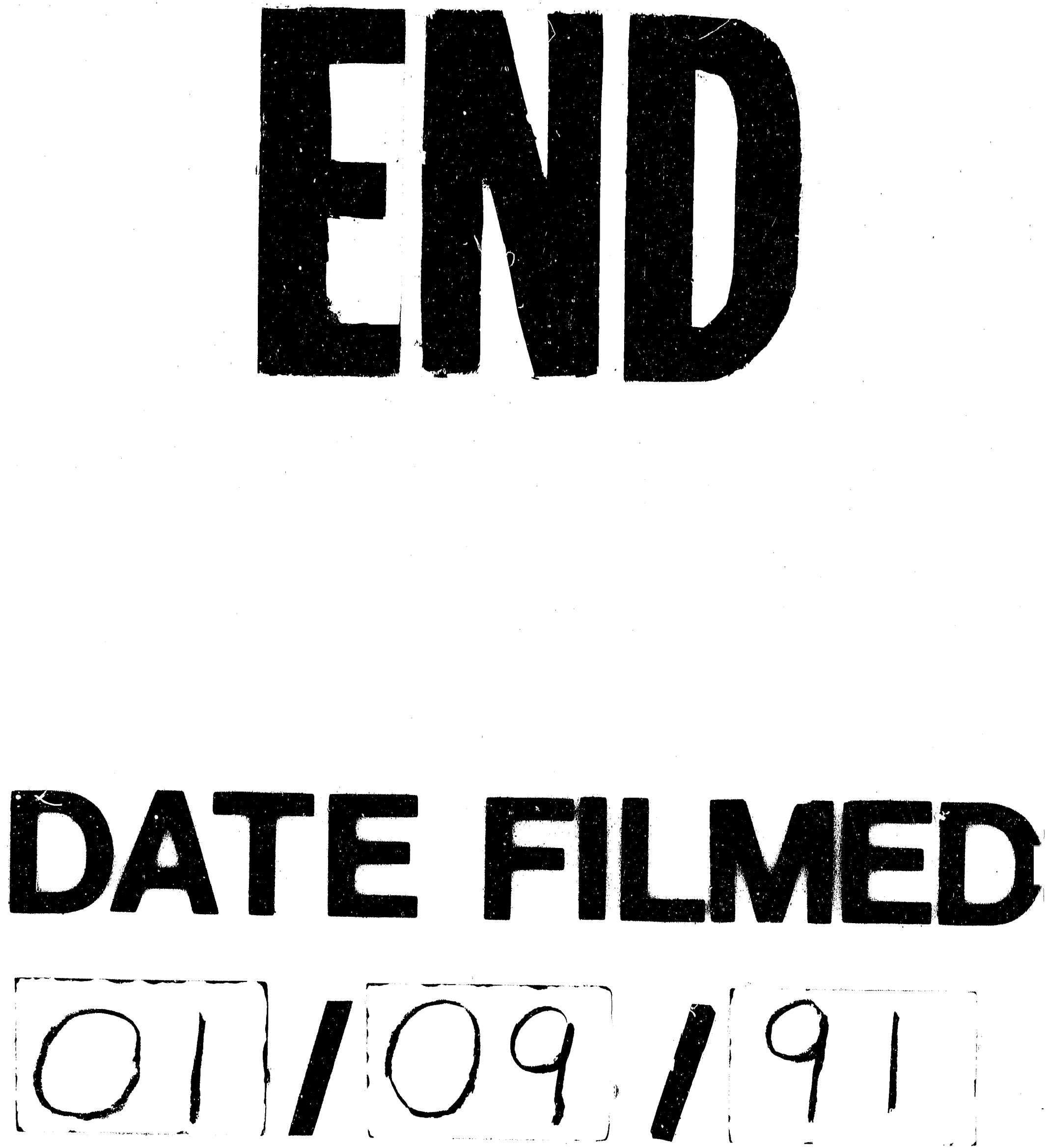
